\title{
Optical nuclei of radio-loud AGN and the Fanaroff-Riley divide ${ }^{\star}$
}

\author{
P. Kharb and P. Shastri
}

\author{
Indian Institute of Astrophysics, Koramangala, Bangalore - 560 034, India \\ e-mail: rhea@iiap.res.in
}

Received 17 June 2003 / Accepted 15 June 2004

\begin{abstract}
We investigate the nature of the point-like optical nuclei that have been found in the centres of the host galaxies of a majority of radio galaxies by the Hubble Space Telescope. We examine the evidence that these optical nuclei are relativistically beamed, and look for differences in the behaviour of the nuclei found in radio galaxies of the two Fanaroff-Riley types. We also attempt to relate this behaviour to the properties of the optical nuclei in their highly beamed counterparts (the BL Lac objects and radio-loud quasars) as hypothesized by the simple Unified Scheme. Simple model-fitting of the data suggests that the emission may be coming from a non-thermal relativistic jet. It is also suggestive that the contribution from an accretion disk is not significant for the FRI objects and for the narrow-line radio galaxies of FRII type, while it may be significant for the Broad-line objects, and consistent with the idea that the FRII optical nuclei seem to suffer from extinction due to an obscuring torus while the FRI optical nuclei do not. These results are broadly in agreement with the Unified Scheme for radio-loud AGNs.
\end{abstract}

Key words. galaxies: active - BL Lacertae objects: general - galaxies: nuclei - quasars: general

\section{Introduction}

The "radio-loud" active galactic nuclei (AGNs) which include radio galaxies, BL Lac objects and quasars, show twin lobes of synchrotron-emitting plasma connected to a "core" by plasma jets on scales of $\sim 100 \mathrm{kpc}$. Fanaroff \& Riley (1974) recognised that the radio morphology of radio galaxies along with their total radio power (at $178 \mathrm{MHz}$ ) fell into two distinct subclasses: the lower-power Fanaroff-Riley type I (FRI) objects show extended plumes and tails with no distinct termination of the jet while the higher-power type II (FRII) objects show narrow, collimated jets and terminal "hotspots". The FRII radio galaxies have systematically more luminous optical emission lines (Zirbel \& Baum 1995) while FRI radio galaxies inhabit richer environments (Prestage \& Peacock 1988); the value of the FRI/FRII radio luminosity break blurs at higher radio frequencies (see Urry \& Padovani 1995), and increases with the optical luminosity of the host galaxy (Ledlow \& Owen 1996). The origin of the $\mathrm{F}-\mathrm{R}$ dichotomy is far from clear: suggested possibilities include differences in the spin of the supermassive black hole resulting in different jet kinetic powers (Baum et al. 1995; Meier 1999), galaxy environments (Smith \& Heckman 1990), and accretion rates (Baum et al. 1995). The dichotomy issue is complicated by the observations of sources having both FRI and FRII characteristics (FRI/II, e.g., Capetti et al. 1995).

AGN jets experience bulk relativistic motion (Blandford \& Konigl 1979) resulting in orientation playing a dominant role

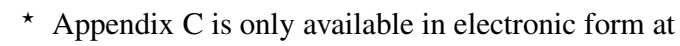
http://www . edpsciences.org in their appearance and a simple Unified Scheme (US) has emerged (e.g., Urry \& Padovani 1995, and refs. therein) according to which the BL Lac objects and radio-loud quasars are the relativistically beamed counterparts of FRI and FRII radio galaxies, respectively. Apart from bulk relativistic motion in radio galaxies, the US requires a ubiquitous optically thick torus in the FRII class of objects (i.e., FRII radio galaxies and radioloud quasars) which hides the powerful optical continuum and broad emission lines from the nucleus in the edge-on objects, while no such torus is required by the US for the FRI class of objects as broad emission lines are weak/absent, and it has not been clear whether or not a torus exists.

The beamed synchrotron emission from the base of the jet or "core" must extend to visible wavelengths and there is strong evidence for it in BL Lacs and quasars (Impey \& Tapia 1990; Wills et al. 1992). Recently, evidence for an optical synchrotron component in the relatively unbeamed radio galaxies has also surfaced, in the form of unresolved nuclear sources in the high resolution images with the Hubble Space Telescope (HST) (e.g., Chiaberge et al. 1999, 2002; Hardcastle \& Worrall 2000; Verdoes Kleijn et al. 2002). These authors argue on the basis of the strong connection with the radio core emission, anisotropy (Capetti \& Celotti 1999) and colour information that these optical nuclei are indeed due to synchrotron radiation.

In this paper, we further test the idea that the unresolved nuclear optical emission from radio galaxies is beamed synchrotron emission from the base of the jet, using the radio core prominence parameter $\left(R_{\mathrm{c}}\right)$ as an indicator of the orientation of the AGN axes. We then attempt to place these correlations in the broader framework of the US and test for consistencies. 
We come up with a model-fitting approach to investigate quantitatively the dependence of the optical emission on orientation and further test the predictions of the US in terms of the presence (or absence) of obscuring tori and the contribution of thermal accretion disks. We list the caveats with regard to our current sample and attempt to address them. The outline of the paper is as follows: in Sect. 2 we discuss the optical nuclei in FRI and FRII radio galaxies and the correlations with $R_{\mathrm{c}}$. In Sect. 3 we compare the optical nuclei with those in BL Lacs and quasars and discuss the results along with modelfitting. The model equations and the fitting procedure are described in Appendices A and B. Section 4 lists the conclusions. Throughout this paper, $H_{0}=75 \mathrm{~km} \mathrm{~s}^{-1} \mathrm{Mpc}^{-1}$ and $q_{0}=0.5$ have been adopted and the spectral index $\alpha$ is defined such that $F_{v}=v^{-\alpha}$.

\section{The optical nuclei in FRI and FRII radio galaxies}

Optical nuclei have been detected in a majority of 3CR, B2 and UGC FRI and FRII radio galaxies with the WFPC2 on board the HST which appear as unresolved sources with angular sizes $\sim 0$.' 1 . The results of studies based on this discovery have been presented by Chiaberge et al. (1999); Capetti \& Celotti (1999); Hardcastle \& Worrall (2000); Capetti et al. (2002); Chiaberge et al. (2002) and Verdoes Kleijn et al. (2002).

For our study, we chose an eclectic sample of FRI and FRII radio galaxies with either such a detected optical nucleus or with an upper limit to its optical flux density from the above-mentioned papers. Our set of FRI radio galaxies comprise 25 3CR (Chiaberge et al. 1999), 17 B2 (Capetti et al. 2002) and 10 UGC FRIs (Verdoes Kleijn et al. 2002) along with NGC 7052 and NGC 6251 from Capetti \& Celotti (1999) and Hardcastle \& Worrall (1999) respectively. Objects with ambiguous morphologies (e.g., FRI/II sources mentioned in Sect. 1) are excluded. So is 3C 386 whose optical "nucleus" is in fact a foreground star (Chiaberge et al. 2002). We thus have 54 FRI radio galaxies spanning a redshift range of $0.0037 \leq z \leq 0.29$. The FRII radio galaxies include $53 \mathrm{ob}-$ jects from the 3CR sample presented in Chiaberge et al. (2002) and 2 B2 FRIIs from Capetti et al. (2002). Among the 55 FRIIs considered, there are 42 narrow-line radio galaxies (NLRGs) and 13 broad-line radio galaxies (BLRGs). The FRII radio galaxies span a redshift range of $0.025 \leq z \leq 0.296$.

Tables C. 1 and C. 2 list the FRI and FRII radio galaxies respectively, along with their optical and radio data. Column 1 lists the IAU name; Col. 2: alternative name; Col. 3: redshift (from the references for radio core data, except for UGC FRIs which are from NED); Col. 4: dust disk minor-to-major axis ratio (superscripts " $d$ " and " $l$ " stand for disk and lane respectively) from Verdoes Kleijn et al. (1999) except 3C 83.1, 3C 296, 3C 449, 3C 465, 3C 326 and 3C 452 which are from de Koff et al. (2000); Col. 5: logarithm of extended radio luminosity at $1.4 \mathrm{GHz}$ in $\mathrm{W} \mathrm{Hz}^{-1}$, calculated using the difference between total and core flux density; data at $5 \mathrm{GHz}$ were converted to $1.4 \mathrm{GHz}$ using $\alpha_{\text {radio }}^{\text {ext }}=0.7$ for extended radio emission; Col. 6: $5 \mathrm{GHz}$ radio core flux density in $\mathrm{mJy}$; Col. 7: reference for the radio core (and total flux density if different); Col. 8: logarithm of radio core prominence standardized to an emitted wavelength of $6 \mathrm{~cm}$; Col. 9: nuclear optical luminosity in $\mathrm{W} \mathrm{Hz}^{-1}$ estimated at an emitted wavelength of $5500 \AA$; Col. 10: reference for nuclear optical flux density/luminosity.

\subsection{The correlations with radio core prominence for radio galaxies}

The radio core prominence parameter, which is the ratio of the core-to-extended radio flux density $\left(R_{\mathrm{c}} \equiv S_{\text {core }} / S_{\text {ext }}\right)$ is a known statistical indicator of orientation (Kapahi \& Saikia 1982; Orr \& Browne 1982) assuming that the core is the unresolved relativistically beamed nuclear jet and the lobes are unbeamed. $R_{\mathrm{c}}$ has indeed been shown to correlate with other orientation-dependent properties both in FRIIs (e.g., Kapahi \& Saikia 1982) and FRIs (e.g., Laing et al. 1999). We use the parameter $R_{\mathrm{c}}$ to test if the luminosities of the optical nuclei $L_{0}$, are orientation-dependent. If the intrinsic optical synchrotron emission from the jet is relativistically beamed by the Doppler factor $\delta$ where $\delta \equiv[\gamma(1-\beta \cos \theta)]^{-1}, \gamma$ being the Lorentz factor $\left(\gamma \equiv 1 / \sqrt{1-\beta^{2}}\right), \beta$ the bulk velocity in units of the speed of light, and $\theta$ being the angle between the radio axis and our line of sight, then $L_{\mathrm{o}}$ should correlate with $R_{\mathrm{c}}$. We note that $L_{\mathrm{o}}$ has been shown to correlate with the radio core luminosity by Chiaberge et al. (1999); Hardcastle \& Worrall (2000).

The optical luminosities of the unresolved HST nuclei were K-corrected and calculated at an emitted wavelength of $5500 \AA$, assuming an optical spectral index $\alpha_{\mathrm{opt}}=1 . R_{\mathrm{c}}$ was calculated using observed radio core and total flux densities at $5 \mathrm{GHz}$ and was further K-corrected to an emitted frequency of $5 \mathrm{GHz}$. For some sources flux densities were estimated from $1.4 \mathrm{GHz}$ assuming $\alpha_{\text {radio }}^{\text {ext }}=0.7$ and $\alpha_{\text {radio }}^{\text {core }}=0$ for the extended and core radio emission, respectively. In Fig. 1 we plot $L_{\mathrm{o}}$ versus $R_{\mathrm{c}}$ for the FRI and FRII radio galaxies.

We note that there are many upper limits to $L_{0}$. We have analysed the statistical significance of the correlations with the aid of the Astronomical Survival Analysis (ASURV) package as implemented in IRAF, which takes into account data which are only upper/lower limits. $L_{\mathrm{o}}$ turns out to be significantly correlated with $R_{\mathrm{c}}$ for the FRI radio galaxies ( $p=0.0001$, generalized Spearman Rank test, see Table 1), arguing that the nuclear optical emission is orientation-dependent in the same sense as the radio emission and may also originate in the relativistically beamed jet. The implication of the above result is consistent with what Verdoes Kleijn et al. (2002) suggest for their UGC FRI sample, viz., that beaming also plays a role in the variance of $L_{0}$, in addition to the intrinsic variance in the nuclear jet $L_{\text {jet }}^{\text {int }}$ which presumably ionizes the line-emitting gas.

For the FRII radio galaxies, the correlation is significant only if the BLRGs (plotted as stars in Fig. 1) are included, while the narrow-line objects do not show a significant correlation by themselves ( $p>0.2$, generalized Spearman Rank test). The narrow-line FRII galaxies show no correlation even with the more sensitive parametric Pearson's correlation test which however uses uncensored data $(p>0.1)$. This lack of correlation could be explained by the presence of a dusty obscuring torus in FRII radio galaxies that is hypothesized by the US; 

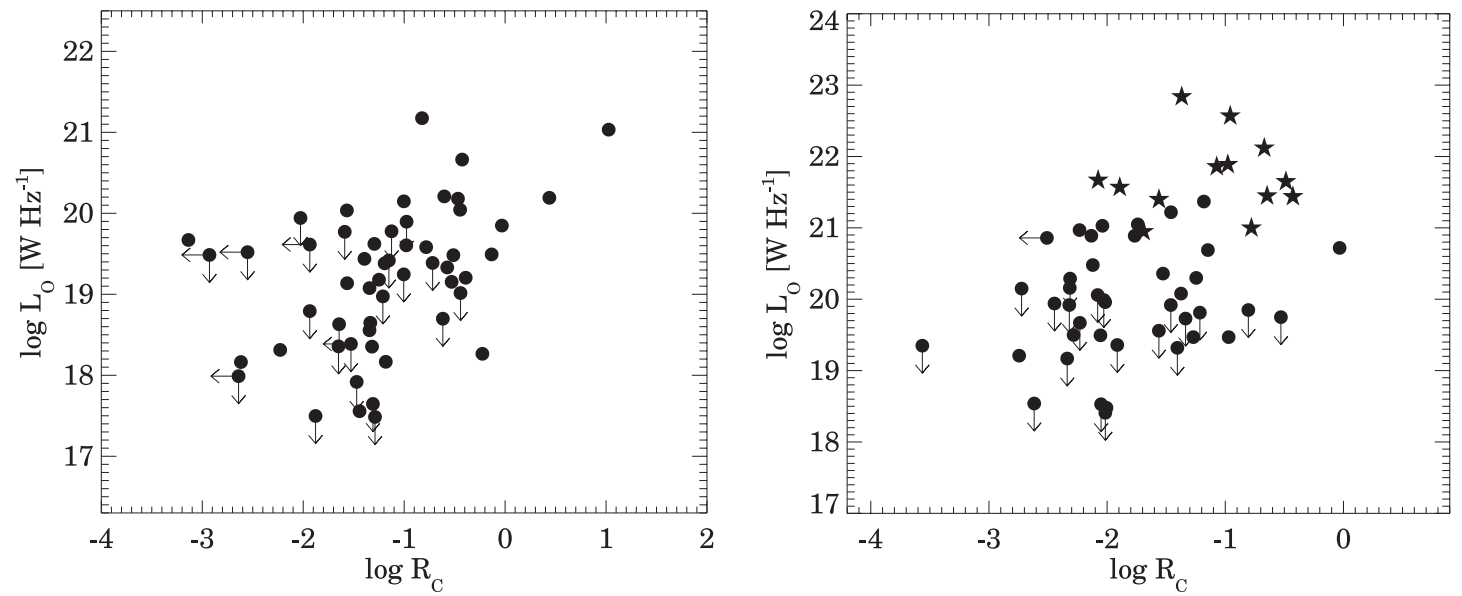

Fig. 1. The luminosity of the optical nuclei $L_{\mathrm{o}}$, plotted against the radio core prominence $R_{\mathrm{c}}$, for the FRI (left) and FRII radio galaxies (right). - radio galaxies, $\star$ BLRGs, $\downarrow$ and $\leftarrow$ upper limits. Statistics for the fits are listed in Table 1 .

Table 1. Statistics of correlations.

\begin{tabular}{|c|c|c|c|c|c|c|}
\hline Type & $N\left(l_{x}, l_{y}\right)$ & $X$ & $Y$ & Spearman & Kendall & Schmitt(slope, intercept) \\
\hline FRI galaxies & $54(5,17)$ & $\log R_{\mathrm{c}}$ & $\log L_{\mathrm{o}}$ & 0.0001 & 0.0001 & $0.52(0.17) \quad 19.40(0.25)$ \\
\hline BL Lacs & $44(6,0)$ & $\log R_{\mathrm{c}}$ & $\log L_{\mathrm{o}}$ & 0.0295 & 0.0323 & $0.17(0.14) \quad 22.45(0.16)$ \\
\hline FRI and BL Lacs & $98(11,17)$ & $\log R_{\mathrm{c}}$ & $\log L_{\mathrm{o}}$ & $<0.0001$ & $<0.0001$ & $1.15(0.10) \quad 20.72(0.14)$ \\
\hline FRI-BL sample & $57(1,2)$ & $\log R_{\mathrm{c}}$ & $\log L_{\mathrm{o}}$ & $<0.0001$ & $<0.0001$ & $1.20(0.17) 20.72(0.16)$ \\
\hline FRII galaxies & $42(1,20)$ & $\log R_{\mathrm{c}}$ & $\log L_{\mathrm{o}}$ & 0.2175 & 0.2392 & $0.32(0.23) \quad 20.12(0.38)$ \\
\hline BLRGs and QSRs & $47(5,0)$ & $\log R_{\mathrm{c}}$ & $\log L_{\mathrm{o}}$ & $<0.0001$ & $<0.0001$ & $0.60(0.12) \quad 23.15(0.12)$ \\
\hline FRII and QSRs & $89(6,20)$ & $\log R_{\mathrm{c}}$ & $\log L_{\mathrm{o}}$ & $<0.0001$ & $<0.0001$ & $1.18(0.11) 22.26(0.17)$ \\
\hline FRII-QS sample & $38(0,3)$ & $\log R_{\mathrm{c}}$ & $\log L_{\mathrm{o}}$ & $<0.0001$ & $<0.0001$ & $1.12(0.11) 22.71(0.15)$ \\
\hline FRI galaxies ${ }^{V}$ & $9(0,2)$ & $b / a(d)$ & $\log L_{\mathrm{o}}$ & $0.2499^{a}$ & 0.2310 & $1.83(1.22) \quad 17.67(0.89)$ \\
\hline$"$ & $14(0,5)$ & $b / a(d+l)$ & $\log L_{\mathrm{o}}$ & $0.0928^{a}$ & 0.0399 & $1.91(0.61) \quad 17.54(0.37)$ \\
\hline$"$ & $9(0,0)$ & $b / a(d)$ & $\log R_{\mathrm{c}}$ & $0.0732^{a}$ & 0.0953 & $-1.09(0.47)-0.19(0.37)$ \\
\hline$"$ & $14(0,0)$ & $b / a(d+l)$ & $\log R_{\mathrm{c}}$ & $0.6065^{a}$ & 0.5200 & $-0.37(0.40)-0.80(0.23)$ \\
\hline FRI galaxies ${ }^{D}$ & $6(0,0)$ & $b / a(d)$ & $\log L_{\mathrm{o}}$ & $0.0845^{a}$ & 0.0909 & $2.24(0.82) \quad 17.62(0.48)$ \\
\hline$"$ & $7(0,0)$ & $b / a(d+l)$ & $\log L_{\mathrm{o}}$ & $0.0543^{a}$ & 0.0509 & $2.33(0.76) \quad 17.63(0.54)$ \\
\hline$"$ & $7^{\star}(0,0)$ & $b / a(d)$ & $\log R_{\mathrm{c}}$ & $0.0802^{a}$ & 0.0985 & $1.39(0.53)-2.30(0.29)$ \\
\hline$"$ & $8^{\star}(0,0)$ & $b / a(d+l)$ & $\log R_{\mathrm{c}}$ & $0.0588^{a}$ & 0.0833 & $1.51(0.48)-2.30(0.29)$ \\
\hline FRI galaxies $^{V+D}$ & $12(0,2)$ & $b / a(d)$ & $\log L_{\mathrm{o}}$ & $0.1855^{a}$ & 0.1531 & $1.53(0.70) \quad 17.95(0.44)$ \\
\hline$"$ & $18(0,5)$ & $b / a(d+l)$ & $\log L_{\mathrm{o}}$ & $0.0353^{a}$ & 0.0248 & $1.90(0.60) \quad 17.66(0.33)$ \\
\hline$"$ & $12(0,0)$ & $b / a(d)$ & $\log R_{\mathrm{c}}$ & $0.7630^{a}$ & 0.6808 & $-0.06(0.69)-1.06(0.49)$ \\
\hline$"$ & $18(0,0)$ & $b / a(d+l)$ & $\log R_{\mathrm{c}}$ & $0.9085^{a}$ & 0.9698 & $0.02(0.48)-1.12(0.28)$ \\
\hline
\end{tabular}

Statistical significance of various correlations (of $X$ and $Y$ ) and linear regression fits. All the results are derived using ASURV as implemented in IRAF. Column 1: the subclass of objects under consideration, "FRII galaxies" refer to narrow-line FRIIs alone, "QSRs" refer to quasars, "FRI-BL" and "FRII-QS samples" refer to the matched subsamples of FRIs and FRIIs as described in Sect. 3.4. FRI galaxies with superscripts $V, D, V+D$ refer to FRI sources from Verdoes Kleijn et al. (1999), de Koff et al. (2000) and from both papers, respectively; $\star$ an additional FRI source $-3 \mathrm{C} 430$ with a disk of $b / a=0.15$ and $\log R_{\mathrm{c}}=-2.5$ was included in the $b / a-\log R_{\mathrm{c}}$ correlation; Col. 2: the number of data points and those with limits in $X$ and $Y$ respectively, in paranthesis; Cols. 3 and 4: the independent and dependent variable respectively; $b / a$ being the ratio of the minor-to-major axis of the extended dust feature seen in the HST images of radio galaxies, " $d$ " and " $l$ " standing for a dust disk and a lane respectively, " $d+l$ " refers to our jointly considering disks and lanes in the correlations; Cols. 5 and 6: probability that no correlation exists between $X$ and $Y$ from Spearman's $\rho$ and Kendall's $\tau$ correlation tests respectively; "a" - Spearman Rank test is not accurate as no. of objects, $N<30$; Col. 7: slope and intercept with standard deviation in parantheses from Schmitt's linear regression test, bootstrap approximation using 200 iterations, $X$ bins $=10, Y$ bins $=10$. 

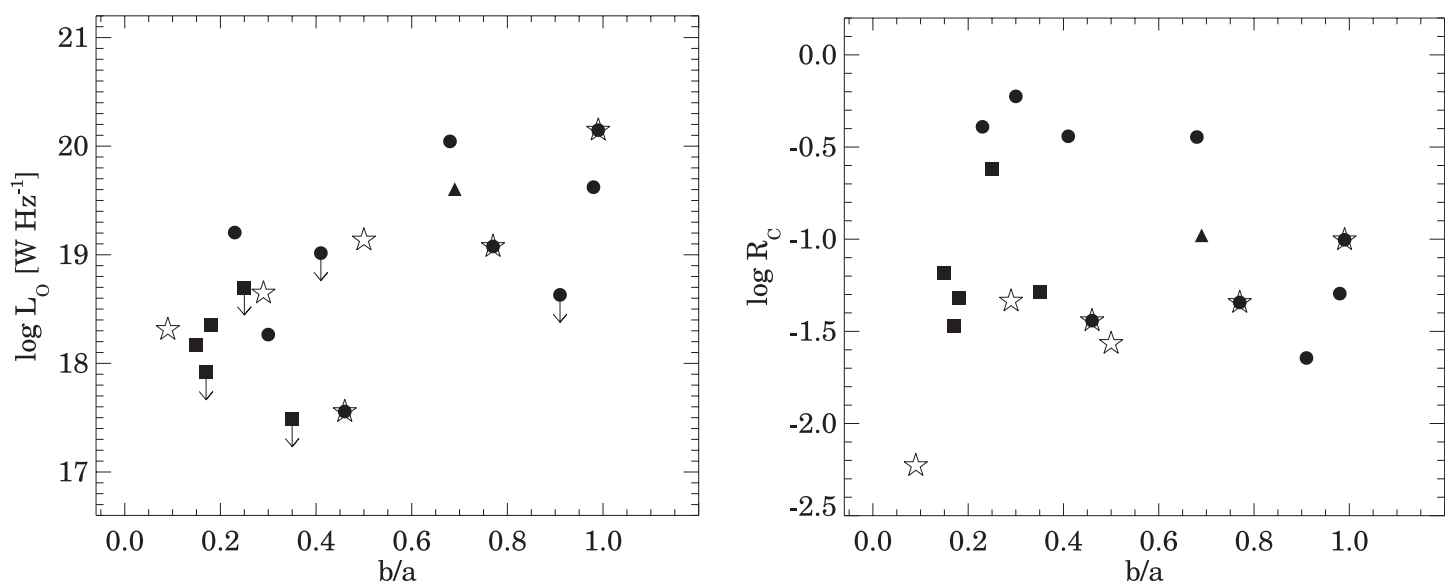

Fig. 2. Nuclear optical luminosity $L_{\mathrm{o}}(l e f t)$ and radio core prominence $R_{\mathrm{c}}$ (right) plotted against minor-to-major axis ratio $b / a$, for the FRI radio galaxies. • and - denote the dust disks and lanes respectively, from Verdoes Kleijn et al. (1999) while the open stars and $\boldsymbol{\Delta}$ denote the dust disks and lanes from de Koff et al. (2000), $\downarrow$ upper limits. The sources common to the two papers are shown by an open star superimposed by $\bullet$. Table 1 lists the statistics for the correlations.

this could also result in the large number of non-detections (also see Chiaberge et al. 2002).

\subsection{Kpc-scale dust disks in FRI radio galaxies}

While the evidence for an obscuring torus in FRIs is so far meagre, much larger dust disks and lanes of sizes $\sim 100 \mathrm{pc}$ to a few kpc have been discovered in many FRI radio galaxies (e.g., Verdoes Kleijn et al. 1999; de Koff et al. 2000). It has been suggested by Verdoes Kleijn et al. (1999), Capetti \& Celotti (1999) and de Koff et al. (2000) that the kpc-scale radio jet tends to align with the axis of this disk. We investigate this point here for the subset of objects where data on dusty disks, as well as $L_{\mathrm{o}}$ and $R_{\mathrm{c}}$ are available. We find different relations of the minor-to-major axis ratio $b / a$, of the extended dust disk with $L_{\mathrm{o}}$ and $R_{\mathrm{c}}$ for the samples presented in the above papers (see Table 1). $b / a$ of the dust disks correlates significantly both with the $L_{\mathrm{o}}$ and $R_{\mathrm{c}}$ for the de Koff et al. FRI galaxies. $b / a$ correlates significantly with $L_{\mathrm{o}}$ for the Verdoes Kleijn et al. FRI sources only when both disks and lanes are considered together. However, they show no correlation with $R_{\mathrm{c}}$ when disks and lanes are taken together, and a correlation in the opposite sense to that predicted, when only disks are considered. When the objects from both samples are combined and both dust disks and lanes are considered, $b / a$ correlates with $L_{\mathrm{o}}$ but not with $R_{\mathrm{c}}$ (see Fig. 2, and Table 1 for the statistical results).

It thus appears that the axes of the extended dust disks do not tend to be aligned with the orientation of the AGN, but that these disks could be causing some extinction of the optical nuclear emission in FRI radio galaxies. This extinction would of course contribute to the scatter in the $L_{\mathrm{o}}-R_{\mathrm{c}}$ correlation.

\section{Comparison with the optical nuclei of the beamed objects and the US}

In the simple US, the beamed counterparts of the FRI and FRII radio galaxies are the BL Lac objects and the radio-loud quasars respectively. In Sect. 2.1 we find the optical emission from galaxy nuclei to be orientation-dependent. Given that optical emission from BL Lacs and quasars is also beamed (e.g., Kapahi \& Shastri 1987; Baker et al. 1994), we attempt to relate the behaviour of the galaxy nuclei to that of BL Lacs and quasars in the framework of the US. We use this framework to extend the correlations of $L_{\mathrm{o}}$ with $R_{\mathrm{c}}$ to higher values of $R_{\mathrm{c}}$. We consider the FRI radio galaxies and BL Lacs together (the "FRI population"), and similarly consider the FRII radio galaxies and radio-loud quasars together (the "FRII population"). To investigate quantitatively the dependence of the optical emission on orientation, we come up with a model-fitting approach. We attempt to apply this to the available data and present the results in Sect. 3.5.1. We further outline the caveats and the drawbacks of our current sample and attempt to address them in Sect. 3.4.

\subsection{The data}

The set of BL Lac objects we considered comprise both radioselected and X-ray selected BL Lacs from Perlman \& Stocke (1993); Vermeulen \& Cohen (1994) and Laurent-Muehleisen et al. (1993), thus including objects having both high and intermediate $R_{\mathrm{c}}$ values. After excluding BL Lacs which showed FRII radio morphology in the form of terminal hotspots viz., 1308+326, 1823+568, 2007+777 (Kollgaard et al. 1992), 1749+701 (O'Dea et al. 1988) and 1803+784 (Cassaro et al. 1999); which were gravitational microlensing candidates, viz., $1413+135$ (this object also has other peculiarities like a spiral host galaxy; Perlman \& Stocke 1993) and which had uncertain redshifts viz., 0716+714, our BL Lac sample consists of 44 objects spanning a redshift range of $0.028 \leq z \leq$ 0.997 . We have considered 34 high $R_{\mathrm{c}}$ radio-loud quasars from Vermeulen \& Cohen (1994) spanning the redshift range of $0.158 \leq z \leq 2.367$.

We have taken the total optical luminosity of BL Lacs and quasars (as derived from their available $V$-band magnitudes) as the nuclear optical luminosity, assuming that the nucleus overwhelms the host galaxy emission. As the BL Lacs are known 

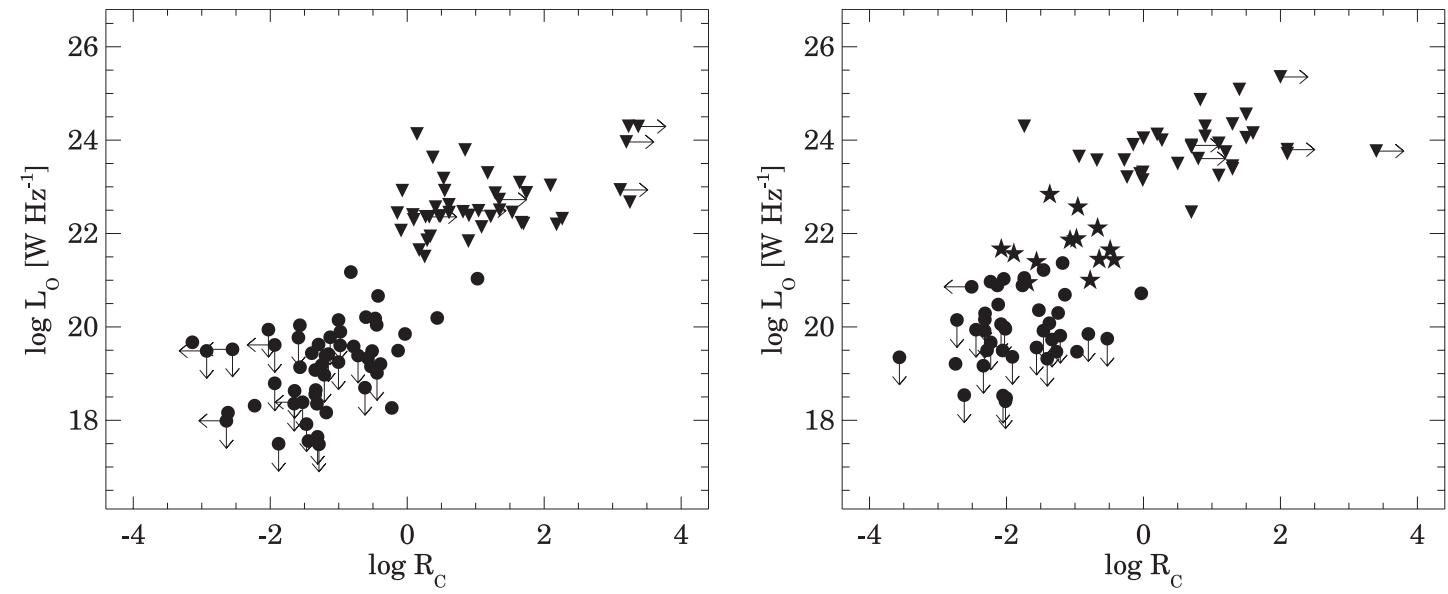

Fig. 3. Nuclear optical luminosity $L_{\mathrm{o}}$ versus radio core prominence $R_{\mathrm{c}}$ for the FRI population $(l e f t): \bullet$ radio galaxies, $\vee$ BL Lac objects. $L_{\mathrm{o}}$ vs. $R_{\mathrm{c}}$ for the FRII population (right): • radio galaxies, $\checkmark$ radio-loud quasars, $\star$ BLRGs. $\downarrow$ and $\leftarrow$ upper limits, $\rightarrow$ lower limits. Statistics for the fits are listed in Table 1.

to be strongly variable, we took radio and optical measurements from the literature that were as closely spaced in time as were available. Several $V$-magnitudes come from optical monitoring campaigns of Pica et al. (1988); Webb et al. (1988) and Falomo et al. (1994). Quasars can also be optically violent variables (OVVs) but they constitute less than $25 \%$ of our quasar sample. The data are tabulated in Tables C.3 and C.4. Column 1 lists the IAU name (B1950); Col. 2: alternative name; Col. 3: redshift (from the references for radio core data, except $1402+042,0333+321,0835+580$ and $0836+710$ which are from Véron-Cetty \& Véron (1998)); Col. 4: V-band magnitude; Col. 5: reference for $m_{v}$; Col. 6: logarithm of extended radio luminosity at $1.4 \mathrm{GHz}$ in $\mathrm{W} \mathrm{Hz}^{-1}$ - taken from the reference for radio core flux density for BL Lacs and calculated using core flux density and radio core prominence for quasars and the BL Lacs $0454+844$ and $0735+178$; data at $5 \mathrm{GHz}$ converted to $1.4 \mathrm{GHz}$ using $\alpha_{\text {radio }}^{\text {ext }}=0.7$ for the extended radio emission; Col. 7: $5 \mathrm{GHz}$ radio core flux density in mJy; Col. 8: reference for the radio core and total flux density (for quasars it is the reference for the radio core flux density and $\log R_{\mathrm{c}}$ ); Col. 9: logarithm of radio core prominence standardized to an emitted wavelength of $6 \mathrm{~cm}$; Col. 10: nuclear optical luminosity in $\mathrm{W} \mathrm{Hz}^{-1}$ estimated at an emitted wavelength of $5500 \AA$. The plots of $L_{\mathrm{o}}$ against $R_{\mathrm{c}}$ for the FRI and FRII populations are shown in Fig. 3.

\subsection{Caveats}

While interpreting the $L_{\mathrm{o}}-R_{\mathrm{c}}$ plots, it is important to keep the following caveats in mind.

1. The objects constitute an eclectic sample, with no rigorous selection criteria applied.

2. The beamed and unbeamed objects are not matched in redshift, nor in extended radio luminosity. We discuss the significance of this in Sect. 3.3 and try to define a "matched" sample in Sect. 3.4.

3. The $L_{\mathrm{o}}$ values for the BL Lacs and quasars are derived from their total magnitudes, and include the host galaxy contribution. Particularly in the intermediate $R_{\mathrm{c}}$ regime for BL Lacs, the host galaxies could contribute significantly to the assumed nuclear optical luminosity.

We address some of these issues later in the paper.

\subsection{Correlations with radio core prominence for the two populations}

For both the FR populations, the $L_{\mathrm{o}}-R_{\mathrm{c}}$ correlation does extend to higher $R_{\mathrm{c}}$, broadly consistent with the predictions of the US and again reinforcing the idea that the optical nuclear emission is orientation-dependent in the same way as the radio core emission and it may thus constitute the optical counterpart of the relativistically beamed radio synchrotron jet. Using survival analysis, the generalized Spearman's Rank correlation test indicates that the FRI and FRII populations both show a significant correlation ( $p<0.0001)$ of $L_{\mathrm{o}}$ with $R_{\mathrm{c}}$ (see Table 1 ).

For the FRII population, we showed in Sect. 2.1 that the narrow-line FRIIs do not show any correlation by themselves. A significant correlation $(p<0.0001$, generalized Spearman Rank test) exists for the broad-line objects, however, i.e., for the the broad-line radio galaxies and quasars. These two observations taken together are consistent with there being obscuration effects by a torus in the FRIIs. Also, though fewer FRIIs than FRIs show detected optical nuclei, optical nuclei have been detected in all the BLRGs observed (where the US predicts no obscuration by the torus), again consistent with this idea. Chiaberge et al. (2000) also suggest obscuration effects on the basis of the non-detection of optical nuclei in some narrow-line FRIIs.

The BL Lacs by themselves also show a significant correlation of $L_{\mathrm{o}}$ with $R_{\mathrm{c}}$, but the plot is flatter than what is expected from beaming alone. This could be due to the fact that their $L_{\mathrm{o}}$ values include the contribution from the host galaxy, particularly since this contamination is likely to be more severe at intermediate values of $R_{\mathrm{c}}$. Although for many BL Lac objects where host galaxies have been imaged (e.g., Jannuzi et al. 1997) the difference between the nuclear and the total optical luminosity $\left(L_{0}\right)$ is less than the $50 \%$ errors assumed in $L_{\mathrm{o}}$ (e.g., Verdoes Kleijn et al. 2002, see Appendix B) for 

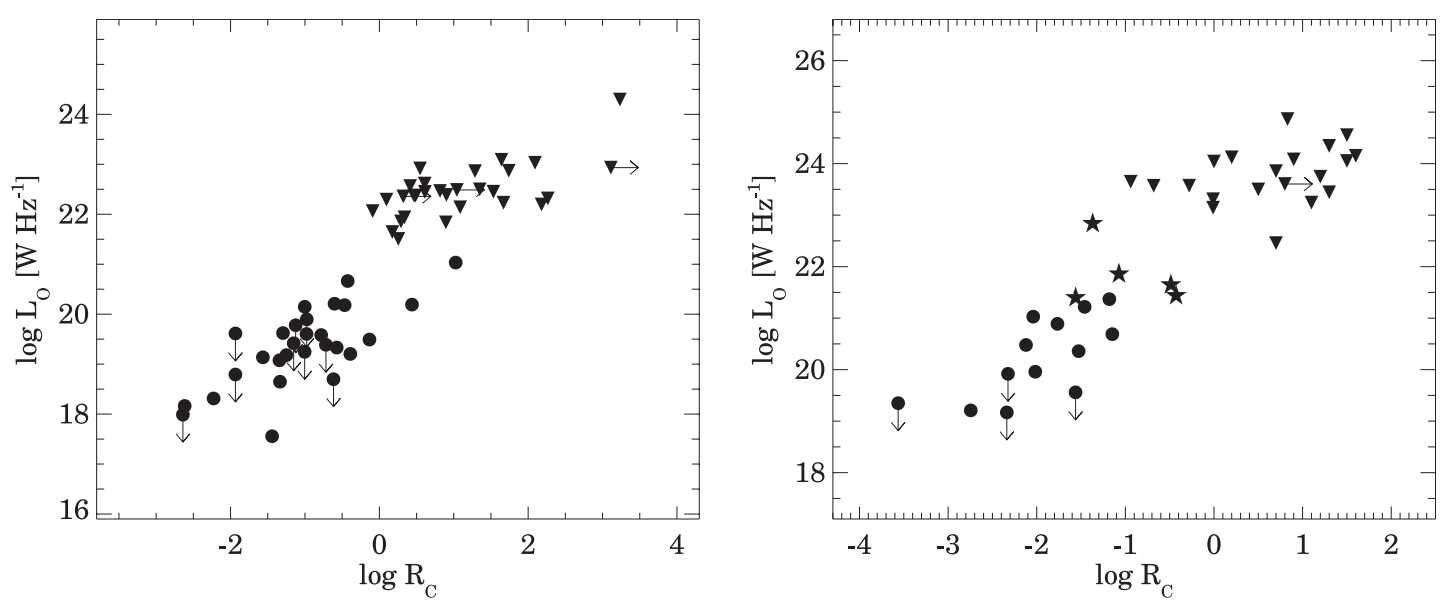

Fig. 4. Nuclear optical luminosity $L_{\mathrm{o}}$ versus radio core prominence $R_{\mathrm{c}}$ for the matched subsample of FRI galaxies and BL Lac objects (left) and FRII galaxies and quasars (right). • radio galaxies, $、$ BL Lac objects (left) and quasars (right), $\star$ BLRGs, $\downarrow$ upper limits, $\rightarrow$ lower limits. Statistics for the fits are listed in Table 1.

some sources this difference can as high as a magnitude (e.g., Kotilainen et al. 1998). In principle, the flattening could also be due to the presence of a luminous accretion disk, in which case the BL Lacs cannot be considered to be consistent with the unbeamed FRI radio galaxies. The use of nuclear luminosities uncontaminated by host galaxy emission for all the objects would clarify the issue. We are in the process of investigating this point which is part of a future paper.

A two-dimensional Kolmogorov-Smirnov test shows that the FRI and FRII populations are different at the $p<$ 0.0001 level. For each of the populations, a multiple linear regression test using the statistics packages STATISTICA and ASURV (the "Buckley James" algorithm) of $R_{\mathrm{c}}$, redshift $(z)$ and extended radio luminosity $\left(L_{\text {ext }}\right)$ as independent variables, shows that the correlation coefficient for the $L_{\mathrm{o}}-R_{\mathrm{c}}$ correlation is the most significant $(p<0.0001) . L_{\mathrm{ext}}$ is the next most significant contributor. Since $L_{\text {ext }}$ can reasonably be assumed to be an indicator of intrinsic AGN power, this implies that variation in intrinsic nuclear power contributes significantly to the scatter in the $L_{\mathrm{o}}-R_{\mathrm{c}}$ correlation. As expected, the nuclear optical luminosity is correlated with redshift, both because luminosity is expected to correlate with redshift, and because of the absence of high redshift radio galaxies in the samples.

\subsection{Matched subsamples of FRI and FRII objects}

Ideally, all the objects in each population ought to be intrinsically similar in the framework of the US, which means that they should all be of similar intrinsic power, from the same volume of space, and with a narrow distribution of other orientationindependent parameters. As a next best step, we attempt here to derive a "matched" sample for the two FR populations, keeping in mind the multiple linear regression results for the whole sample discussed in the previous section.

For the FRI matched subsample, we restrict the redshifts to $z<0.3$ and the extended radio luminosity at $1.4 \mathrm{GHz}$ to $23.5 \leq \log L_{\mathrm{ext}} \leq 25 \mathrm{~W} \mathrm{~Hz}^{-1}$. For the FRII matched subsample, the redshifts are constrained to $z<1.3$, while the extended radio luminosity is $26.2 \leq \log L_{\text {ext }} \leq 27.6 \mathrm{~W} \mathrm{~Hz}^{-1}$. Figure 4 shows the $L_{\mathrm{o}}-R_{\mathrm{c}}$ correlations for these subsamples while the correlation and regression parameters are listed in Table 1. We find that the scatter seen in Fig. 3 is considerably reduced in Fig. 4 and the correlations improve significantly compared to the unrestricted samples. Multiple linear regression tests on the restricted samples with the independent variables, $R_{\mathrm{c}}, z, L_{\mathrm{ext}}$ show that the $L_{\mathrm{o}}-R_{\mathrm{c}}$ is still the strongest correlation $(p<0.0001)$ while the contribution of $L_{\text {ext }}$ is no longer significant.

\subsection{Model-fitting the $L_{\mathrm{o}}-R_{\mathrm{c}}$ data}

If bulk relativistic motion with a single Lorentz factor $(\gamma)$ value applicable to the whole population were alone responsible for the variation in $L_{\mathrm{o}}$, then the logarithmic plot of $L_{\mathrm{o}}$ against $R_{\mathrm{c}}$ would be linear. Any additional factors such as orientation effects due to a torus or thin thermal disk will cause this relationship to deviate from linearity. We attempt to fit some simple models to the data along these lines. Appendix A gives the model equations while Appendix B describes the different models considered along with the model-fitting procedure.

We assume that the nuclear optical luminosity $L_{\mathrm{o}}$ is, in the most general case, due to the sum of synchrotron emission from the base of a relativistic jet, and thermal emission from a thin accretion disk, modified by the presence of an optically thick torus. Keeping in mind that we are only attempting to explore the potential of such a model-fitting approach and that our sample is not rigorously selected, we consider this simple model here and do not include the possibility of variation in intrinsic nuclear power as discussed in Sect. 3.3, nor the possibility of extinction of the optical nucleus by an extended kpc-scale dusty disk which was discussed in Sect. 2.2. Our models also do not take into account any intrinsic spread in the Lorentz factors, nor the possibility that the relevant Lorentz factor for the highly beamed and mildly beamed subclasses may be systematically different due to a "spine-sheath" type structure of the jet (e.g., Hardcastle et al. 1996; Laing et al. 1999). However, as the multiple linear regression tests discussed in Sect. 3.3 
Table 2. Parameters from the different model-fits for the FR populations.

\begin{tabular}{|c|c|c|c|c|c|c|c|c|}
\hline & & & & & Model & Outputs & & \\
\hline & Model & $A_{V_{0}}$ & $\theta_{\mathrm{c}}$ & $L_{\text {jet }}^{\mathrm{int}}$ & $\sigma$ & $L_{\text {disk }}$ & $\sigma$ & AIC \\
\hline \multirow{5}{*}{ FRI } & Jet only & $\ldots$ & $\ldots$ & $2.7 e+20$ & $1.3 e+19$ & $\ldots$ & $\ldots$ & 295.2 \\
\hline & Jet+Disk & $\ldots$ & $\ldots$ & $2.6 e+20$ & $1.4 \mathrm{e}+19$ & $8.5 e+17$ & $1.1 \mathrm{e}+18$ & 297.1 \\
\hline & Jet+Disk+Torus & 3.0 & 45 & $2.7 e+20$ & $1.5 \mathrm{e}+19$ & $3.5 e+18$ & $1.6 e+18$ & 304.5 \\
\hline & Jet+Torus & 3.0 & 45 & $2.9 \mathrm{e}+20$ & $1.4 \mathrm{e}+19$ & $\ldots$ & $\ldots$ & 303.1 \\
\hline & Best fit & 0.1 & 90 & $2.6 e+20$ & $1.3 e+19$ & $\ldots$ & $\ldots$ & 295.3 \\
\hline \multirow{4}{*}{ Matched FRI } & Jet only & $\ldots$ & $\ldots$ & $3.8 \mathrm{e}+20$ & $2.5 e+19$ & $\ldots$ & $\ldots$ & 132.3 \\
\hline & Jet+Disk & $\ldots$ & $\ldots$ & $4.1 \mathrm{e}+20$ & $2.9 \mathrm{e}+19$ & $-7.9 e+18$ & $2.0 \mathrm{e}+18$ & 133.4 \\
\hline & Jet+Disk+Torus & 3.0 & 45 & $4.4 \mathrm{e}+20$ & $3.3 e+19$ & $-1.4 \mathrm{e}+18$ & $4.1 \mathrm{e}+18$ & 137.0 \\
\hline & Jet+Torus & 3.0 & 45 & $4.4 \mathrm{e}+20$ & $2.9 e+19$ & $\ldots$ & $\ldots$ & 135.0 \\
\hline \multirow{5}{*}{ FRII } & Jet only & $\ldots$ & $\ldots$ & $5.8 \mathrm{e}+21$ & $3.0 \mathrm{e}+20$ & $\ldots$ & $\ldots$ & 269.6 \\
\hline & Jet+Disk & $\ldots$ & $\ldots$ & $6.5 \mathrm{e}+21$ & $3.7 e+20$ & $-6.7 e+19$ & $1.2 \mathrm{e}+19$ & 270.7 \\
\hline & Jet+Disk+Torus & 3.0 & 45 & $6.7 e+21$ & $3.9 e+20$ & $-5.8 \mathrm{e}+19$ & $1.5 \mathrm{e}+19$ & 272.9 \\
\hline & Jet+Torus & 3.0 & 45 & $6.1 e+21$ & $3.2 \mathrm{e}+20$ & $\ldots$ & $\ldots$ & 271.5 \\
\hline & Best fit & 3.0 & 37 & $6.5 e+21$ & $3.4 \mathrm{e}+20$ & $\ldots$ & $\ldots$ & 270.8 \\
\hline \multirow{4}{*}{ Matched FRII } & Jet only & $\cdots$ & $\ldots$ & $1.6 e+21$ & $1.3 e+20$ & $\ldots$ & $\ldots$ & 94.3 \\
\hline & Jet+Disk & $\cdots$ & $\cdots$ & $1.8 \mathrm{e}+21$ & $1.5 \mathrm{e}+20$ & $-1.9 \mathrm{e}+20$ & $2.1 \mathrm{e}+19$ & 93.4 \\
\hline & Jet+Disk+Torus & 3.0 & 45 & $2.0 \mathrm{e}+21$ & $1.7 \mathrm{e}+20$ & $-2.1 e+20$ & $2.5 e+19$ & 95.9 \\
\hline & Jet+Torus & 3.0 & 45 & $1.8 \mathrm{e}+21$ & $1.4 \mathrm{e}+20$ & $\ldots$ & $\ldots$ & 96.2 \\
\hline \multirow[t]{2}{*}{ BL FRII } & Jet only & $\ldots$ & $\ldots$ & $2.2 \mathrm{e}+22$ & $1.6 \mathrm{e}+21$ & $\ldots$ & $\ldots$ & 127.3 \\
\hline & Jet+Disk & $\ldots$ & $\ldots$ & $1.3 e+22$ & $1.2 \mathrm{e}+21$ & $1.5 \mathrm{e}+22$ & $2.7 \mathrm{e}+21$ & 123.3 \\
\hline \multirow[t]{2}{*}{ BL Lacs` } & Jet only & $\ldots$ & $\ldots$ & $1.2 \mathrm{e}+21$ & $9.0 e+19$ & $\ldots$ & $\ldots$ & 118.4 \\
\hline & Jet+Disk & $\ldots$ & $\ldots$ & $6.2 \mathrm{e}+19$ & $1.4 \mathrm{e}+19$ & $3.3 e+22$ & $3.0 \mathrm{e}+21$ & 78.6 \\
\hline
\end{tabular}

$\star$ See Sect. 3.5.1 in the text. In Col. 1 FRI/FRII and Matched FRI/FRII stand for the FRI/FRII population and it's matched subsample as discussed in Sect. 3.4, BL FRII stand for the broad-line FRIIs viz., BLRGs and quasars. $A_{V_{0}}$ and $\theta_{\mathrm{c}}$ (in degrees) are the fixed initial parameters for the models where a torus is incorporated. $\sigma$ is the standard deviation for the variable on the left. A lower AIC (Akaike's Information Criterion) value indicates a better model fit. The "Best fit" model is the "Jet+Torus" model for FRIs and FRIIs for which AIC is lowest (see Appendix B and Sect. 3.5.1), the $A_{V_{0}}$ and $\theta_{\mathrm{c}}$ (in degrees) are the parameters corresponding to this fit.

suggest, orientation appears to play the most dominant role in the variation of $L_{\mathrm{o}}$. To quantify the goodness-of-fit of a particular model, we have used Akaike's information criterion (AIC). The AIC (Akaike 1974) is a likelihood criterion with an added penalty term corresponding to the complexity of the model, and measures the trade-off between model complexity/parsimony and goodness-of-fit. Smaller AIC values indicate a better fit. Appendix B describes the usage of AIC to derive the best model-fits to the FRI and FRII data.

\subsubsection{The results}

The results of the model-fitting are given in Table 2. For the FRI population, the "Jet-only" model is better than all the others. The "Jet+Disk" model in fact yields a value for the accretion disk luminosity $L_{\text {disk, }}$, which is comparable to its standard deviation $\sigma$ obtained from the fitting. Further, the "Jet+Torus" model is best fitted by the torus extinction parameter $A_{V_{0}} \approx 0.1$ and half-opening angle $\theta_{\mathrm{c}} \approx 90^{\circ}$, which are equivalent to there being no torus. We note here that, based on the high rate of detections of optical nuclei in FRIs, Chiaberge et al. (1999) have also suggested that there is no obscuring torus in them. Further, Perlman et al. (2001) have failed to detect thermal emission from a dusty torus in the $10 \mu \mathrm{m}$ image of the nearby FRI radio galaxy, M 87.

For the FRII population as a whole, the results are less clear. Formally, the "Jet-only" model has the lowest AIC value, but the other models also yield comparable values. However, the $L_{\text {disk }}$ that is obtained for "Jet+Disk" and "Jet+Disk+Torus" is unphysical. When only the broad-line objects among the FRIIs, viz., the BLRGs and quasars are considered, the "Jet+Disk" model was a better fit than the "Jet-only" model (Table 2). This is consistent with the fact that the "big blue bump" (attributed to the accretion disk) is observed in all these objects, and, in the framework of the US, their central regions are not obscured by the torus. For the whole population, a larger number of data points in the regime where the disk is expected to be relatively most prominent, viz., the intermediate 

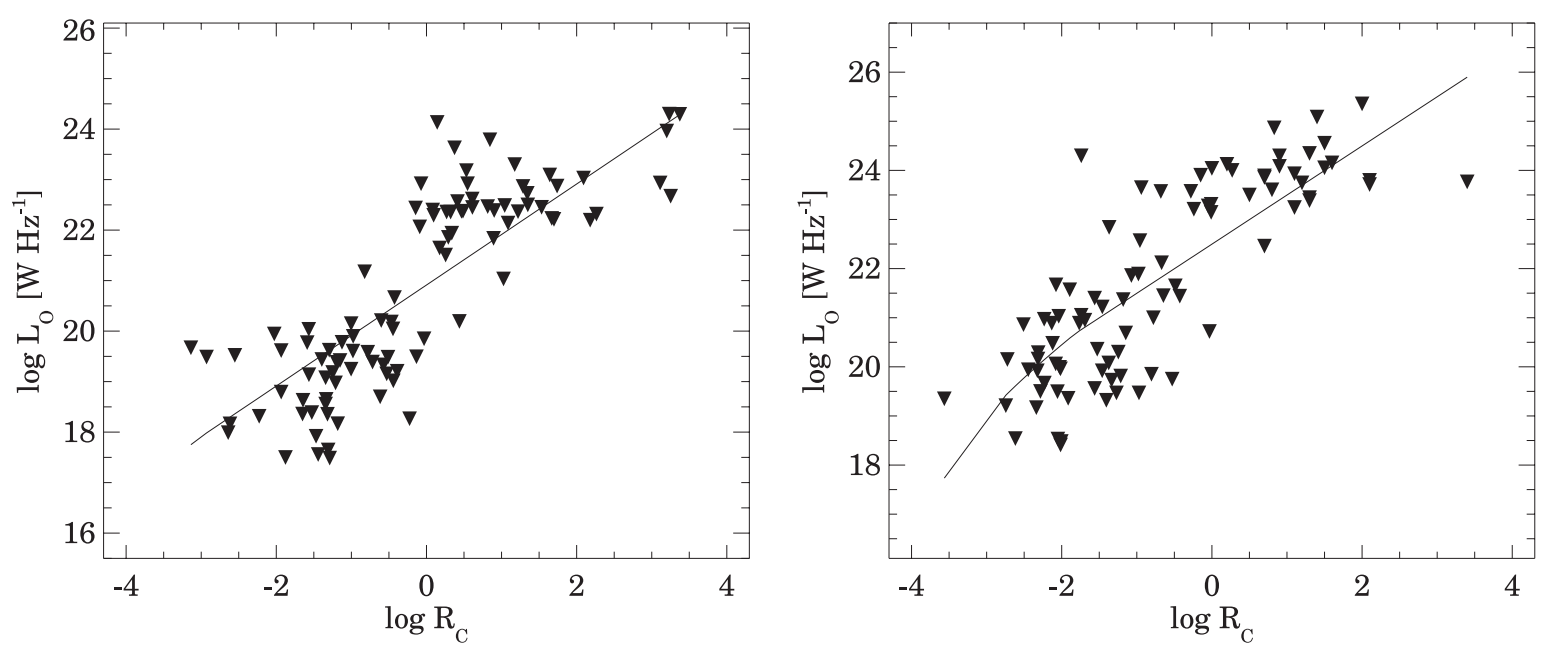

Fig. 5. Best fits to the FRI (left) and FRII (right) populations using only a "Jet+Torus" model. Table 2 lists the model parameters.
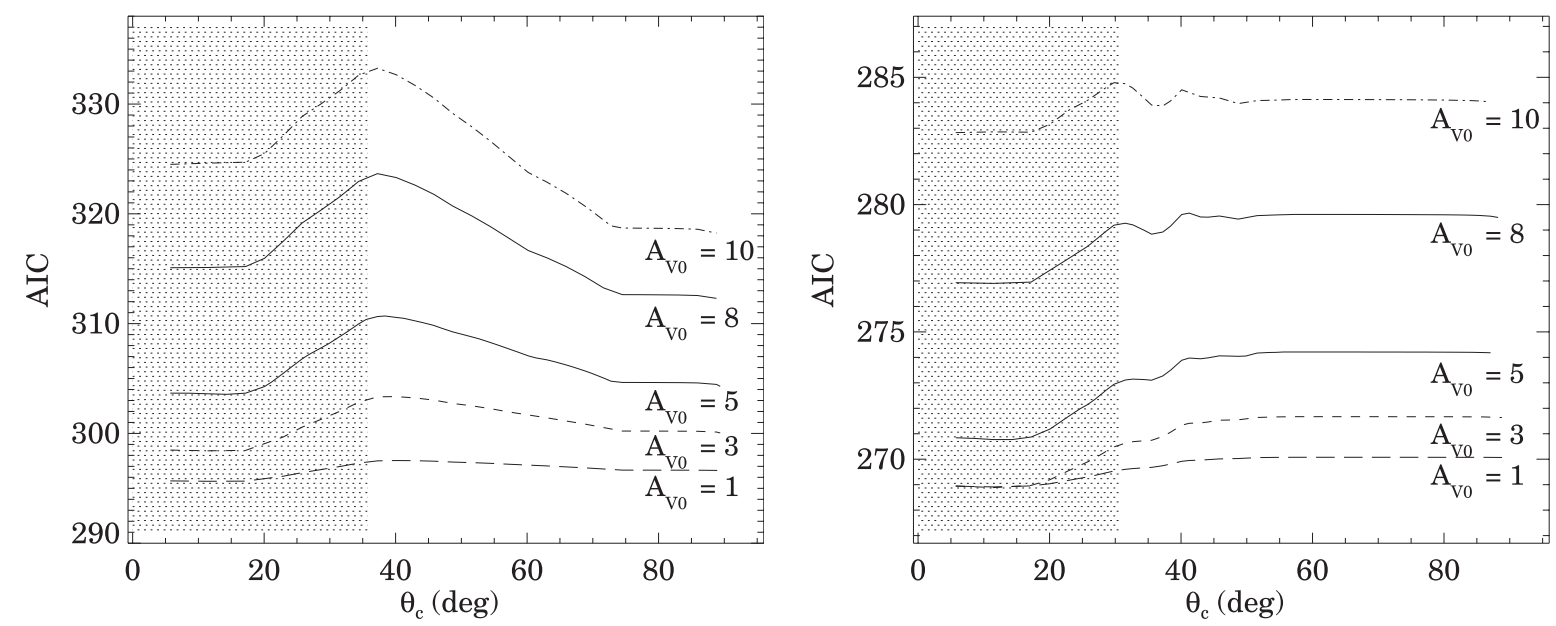

Fig. 6. AIC values for different initial $A_{V_{0}}$ plotted against torus opening angles $\theta_{\mathrm{c}}$ (in degrees) for the FRI (left) and the FRII (right) populations for the "Jet+Torus" model. For a given $A_{V_{0}}$, AIC was estimated at $2^{\circ}$ intervals of $\theta_{\mathrm{c}}$. The shaded area denotes the region where the model becomes unphysical (see Sect. 3.5.1). In the physical regime, AIC reaches a minimum at around $90^{\circ}$ for the FRIs and $37^{\circ}$ for the FRIIs.

$R_{\mathrm{c}}$ region, is required to derive a more robust quantitative value for $L_{\text {disk }}$ since at large $R_{\mathrm{c}}$ the jet overwhelms the disk emission and at very small $R_{\mathrm{c}}$ the torus obscures it. Table 2 lists the fitted parameters of the "Best fit" model for both the FR populations and Fig. 5 shows the best fit curves to each of the FR populations.

It is interesting here, that the "Jet-only" model is unambiguously the best-fit for the FRIs, whereas, several models give comparable fits to the FRIIs. Given this difference in the behaviour of the two classes, we carry the procedure a bit further by contrasting the behaviour of the AIC for the FRIs and FRIIs in the "Jet+Torus" model case. In Fig. 6 we plot the AIC against the torus half-opening angle for different fixed values of $A_{V_{0}}$. Figure 6 shows that the families of AIC plots for the two populations differ systematically from each other. The plots can be broadly divided into two parts. Below $\theta_{\mathrm{c}} \approx 30^{\circ}$ the AIC drops for both the FRI and FRII population (shaded region in Fig. 6). This formally implies that the fit gets better for opening angles of the torus that are smaller than $\theta_{\mathrm{c}} \approx 30^{\circ}$, but clearly is the result of the algorithm trying to fit the entire variation in $L_{\mathrm{o}}$ by torus obscuration alone. For the FRIs, above $\theta_{\mathrm{c}} \approx 35^{\circ}$, the AIC declines again and reaches a minimum at angles close to $90^{\circ}$, consistent with there being no torus. For the FRIIs, on the other hand the AIC does not decline appreciably above $\theta_{\mathrm{c}} \approx 35^{\circ}$. In addition it shows a conspicuous minimum at $\theta_{\mathrm{c}} \approx 37^{\circ}$.

Thus although simple model-fitting using the LM algorithm yields ambiguous results for the FRIIs on the face of it, by rejecting the possibility of the entire variation in $L_{\mathrm{o}}$ being due to obscuration by a torus we obtain model parameters that are broadly consistent with the predictions of the US. The modelfitting results in the best $\theta_{\mathrm{c}}$ roughly coinciding with the angle where the upper limits to $L_{\mathrm{O}}$ start appearing in FRII galaxies. The best $A_{V_{0}}$ turns out to $\approx 3$ mag for the FRIIs at the best $\theta_{\mathrm{c}}$ while for the FRIs, $A_{V_{0}}$ turns out to be $\approx 0$. We point out that the $A_{V_{0}}$ that we infer here is of the nature of a lower limit, since detection limits would exclude measured data points corresponding to higher values of $A_{V_{0}}$. We find that the behaviour of FRI and FRII optical nuclei is distinctly different in that the model-fitting results are unambiguous for the FRIs while they are not so for the FRIIs, hinting at intrinsic differences between FRIs and FRIIs. 

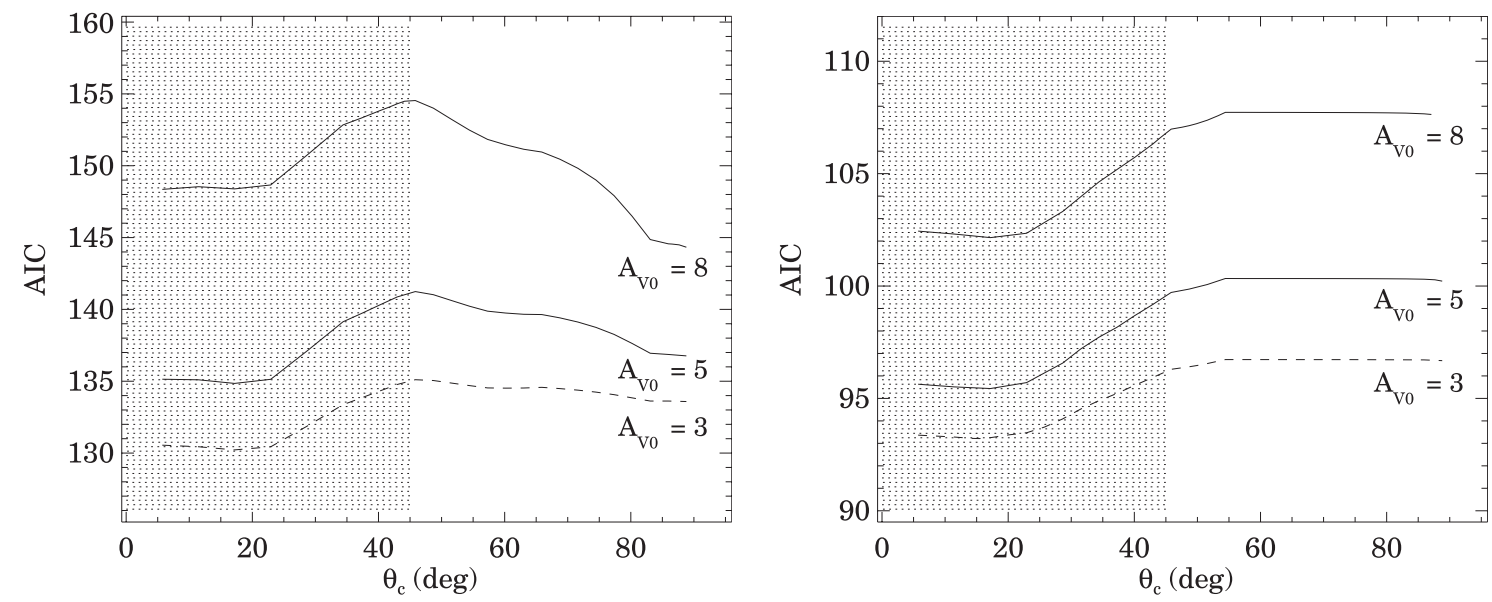

Fig. 7. AIC values for different initial $A_{V_{0}}$ plotted against torus opening angles $\theta_{\mathrm{c}}$ (in degrees) for the matched subsamples of FRI (left) and the FRII (right) populations for the "Jet+Torus" model. The shaded area denotes the region where the model becomes unphysical (see Sect. 3.5.1). In the physical regime, AIC reaches a minimum at around $90^{\circ}$ for the FRIs while the case is not clear for the FRIIs.

It may be recalled that the BL Lac objects taken by themselves show a flatter logarithmic distribution of $L_{\mathrm{o}}$ against $R_{\mathrm{c}}$ than would be expected from beaming alone (Sect. 3.3), and indeed model fitting just the BL Lacs gives the "Jet+Disk" model to be the best one for them, with an implied $L_{\text {disk }}$ of $3.3 \times 10^{22} \mathrm{~W} \mathrm{~Hz}^{-1}$ (see Table 2). As was stated in Sect. 3.3, this is most likely to be due to the contaminating host galaxy luminosity mimicking emission from a disk. We note however, that unless values for their optical nuclei are used that are uncontaminated by the host galaxy, we cannot totally rule out an accretion disk in the BL Lacs, and this implies that they may not be intrinsically similar to the FRI radio galaxies.

For the smaller matched subsamples of FRIs and FRIIs, the variation of AIC with $\theta_{\mathrm{c}}$ (see Fig. 7) is broadly similar to that of their respective unrestricted samples. This validates our procedure and the qualitative results for the whole sample regarding the differences between the FRIs and FRIIs. The only discernible difference for the "matched" FRIIs is that the "AIC minimum" observed at $\theta_{\mathrm{c}} \approx 37^{\circ}$ for the whole sample, is now no longer prominent. However, this may not be surprising in view of the fact that the "matched" FRII objects are much fewer in number, especially at low $R_{\mathrm{c}}$ values.

We note that for neither the FRIs nor FRIIs is there any correlation between the residuals of the model-fit with $R_{\mathrm{c}}$ and $L_{\mathrm{o}}$. However, there seems to be a weak correlation for the BL Lacs considered alone. This may reflect the effects of ignoring the host galaxy luminosity when taking the nuclear optical luminosity.

We note that while reliable quantitative results cannot be obtained using the current data because of the drawbacks in the sample, the approach and the results indicate that it is a good approach to derive various parameters if data for a rigorous and large sample are available. Better data would allow more parameters to be incorporated and controlled.

\subsubsection{The bulk Lorentz factor}

As an approximation we assume that a single $\gamma$ value is applicable to each population, and that all orientations are represented in each population, and therefore that the minimum and maximum values of $R_{\mathrm{c}}$ in each population correspond to orientations perpendicular and parallel to our line of sight respectively. The formula using the $R_{\mathrm{c}}^{\min }$ and $R_{\mathrm{c}}^{\max }$ (Eq. (A.3) in the Appendix) results in the lower limit to the maximum Lorentz factor $\gamma_{\max } \approx 9.7$ for the FRI population. For the FRII population we get a value of $\gamma_{\max } \gtrsim 11.5$, obtained using a quasar with a upper limit to its extended radio emission, and therefore the actual lower limit to $\gamma_{\max }$ could be higher. These values broadly agree with those obtained by Urry \& Padovani (1995): $\gamma_{\max } \gtrsim 9$ for the FRI population and $\gtrsim 13$ for the FRII population assuming $p=3$. However, based on the correlation of optical and radio core emission with the isotropic $\mathrm{H} \alpha+[\mathrm{NII}]$ emission, Verdoes Kleijn et al. (2002) have obtained a constraint on the value of $\gamma$ of $\$ 2$ (assuming $p=3$ ), albeit for FRI radio galaxies alone.

\section{Conclusions}

We use the radio core prominence $R_{\mathrm{c}}$ as a statistical indicator of orientation and find that the systematic differences between radio-loud AGN of the two Fanaroff-Riley types appear to also extend to their optical nuclei, in a manner that is consistent with the predictions of the simple US. We find that

1. The luminosity of the pc-scale optical nuclei in the FRI radio galaxies is orientation-dependent, while that in the FRII radio galaxies is not. This result is consistent with the idea that FRIIs contain an obscuring torus, (as required by the simple US) whereas there is no torus in the FRIs.

2. For the FRI radio galaxies, though the correlation with orientation is very significant, there remains considerable residual scatter. This may be due to obscuration from an extended kpc-scale dusty disk. The axis of this disk appears unrelated to the AGN axis. The residual scatter may also be due to intrinsic variation in the optical luminosity.

3. The nuclear optical luminosity correlates significantly with $R_{\mathrm{c}}$, or, equivalently orientation, for the FRI radio galaxies and BL Lacs of FRI morphology taken 
together - the FRI population. Our model-fitting suggests that a relativistically beamed optical jet gives the best fit.

4. For the FRII radio galaxies and radio-loud quasars taken together - the FRII population, the nuclear optical luminosity again correlates significantly with $R_{\mathrm{c}}$. Our model-fitting indicates that formally the best fit is again a beamed synchrotron jet. But a beamed jet obscured by a torus with an inferred opening angle close to $40^{\circ}$ is a comparable fit, and is able to explain the contrasting behaviour of the FRI and FRII data.

5. The scatter in the $L_{\mathrm{o}}-R_{\mathrm{c}}$ correlation for both the FR populations is likely to be primarily due to the spread in intrinsic AGN power, although extended dusty disks may also contribute to the scatter in FRI radio galaxies.

6. Our model-fitting suggests that the luminosity of the intrinsic (i.e., unbeamed) jet in the FRIIs is approximately an order of magnitude larger than for the FRIs, although this result needs to be confirmed using a rigorous sample.

7. The data for the broad-line FRIIs alone are fitted best by a model that comprises a relativistic jet and a geometrically thin optically thick disk, consistent with the presence of the "big blue bump" in them.

The robustness of the above results is limited by the facts that (a) the "samples" used are eclectic; (b) the luminosities of the optical nuclei in the highly beamed objects are contaminated by the contributions of the host galaxies; and (c) there could be variability between the epochs of the optical and radio measurements. A robust analysis requires rigorous measurement of the optical luminosity as well as samples that are rigorously selected, with the objects of a given FR population chosen to be intrinsically similar in the framework of the US and from the same volume of space.

Acknowledgements. We are very grateful to Prof. Thriyambakam Krishnan for his scrutiny of our statistical tests and results, useful suggestions and extensive discussions. We also thank the anonymous referee for comments that improved the paper.

\section{Appendix A: The model equations}

We give here the equations that form the basis of our modelfitting procedure. In the most general case, we assume that the nuclear optical luminosity $L_{\mathrm{o}}$ is due to the sum of synchrotron emission from the base of a relativistic jet, and thermal emission from a thin accretion disk, modified by the presence of an optically thick torus. We write,

$L_{\mathrm{o}}=\left(\delta^{p} L_{\mathrm{jet}}^{\mathrm{int}}+L_{\mathrm{disk}} \cos \theta\right) \times 10^{-A_{V} / 2.5}$

$L_{\text {jet }}^{\text {int }}$ is the intrinsic synchrotron luminosity from the base of the jet which is relativistically beamed by the factor $\delta^{p}$, where $\delta$ is the Doppler factor and for a jet spectral index of $\alpha$ the jet structure parameter $p$ is given by $2+\alpha$ or $3+\alpha$ depending on whether the jet is continuous or blobby (e.g., Urry \& Padovani 1995). $L_{\text {disk }}$ is the luminosity of a geometrically thin optically thick accretion disk, whose apparent luminosity is orientationdependent due to projection (the $\cos \theta$ term). $A_{V}$ is the extinction resulting from the torus in the $V$ band. For a halfopening angle of the torus $\theta_{c}$, we have,

$\begin{array}{ll}A_{V}=A_{V_{0}}\left(1-\frac{\cos \theta}{\cos \theta_{\mathrm{c}}}\right) & \text { for } \theta \geq \theta_{\mathrm{c}} \\ A_{V}=0 & \text { for } \theta<\theta_{\mathrm{c}}\end{array}$

(Simpson 1996).

Thus, for $\theta=90^{\circ} A_{V}=A_{V_{0}}$.

For the Lorentz factor of bulk relativistic motion of the nuclear jet (e.g., Appendix C, Urry \& Padovani 1995) we have,

$\gamma=\left(\frac{1}{2^{p-1}} \frac{R_{\mathrm{c}}^{\max }}{R_{\mathrm{c}}^{\min }}\right)^{\frac{1}{2 p}}$,

$R_{\mathrm{c}}^{\mathrm{int}}=\frac{\gamma^{p} R_{\mathrm{c}}^{\min }}{2}$

where $R_{\mathrm{c}}^{\min }$ and $R_{\mathrm{c}}^{\max }$ are the minimum and maximum values of $R_{\mathrm{c}}$, i.e., the values of $R_{\mathrm{c}}$ at edge-on $\left(\theta \sim 90^{\circ}\right)$ and pole-on $\left(\theta \sim 0^{\circ}\right)$ inclinations of the AGN respectively, and $R_{\mathrm{c}}^{\mathrm{int}}$ is the intrinsic flux density ratio of the core and the extended radio emission. We now obtain the orientation to the line of sight, $\theta$, in terms of $R_{\mathrm{c}}$. We use the relativistic beaming formulae which take into account contributions from both the approaching and receding jet (e.g., Urry \& Padovani 1995).

$R_{\mathrm{c}}=R_{\mathrm{c}}^{\mathrm{int}}\left[\frac{1}{[\gamma(1-\beta \cos \theta)]^{p}}+\frac{1}{[\gamma(1+\beta \cos \theta)]^{p}}\right]$.

We assume a value of 3 for the jet structure factor $p$. We note that Urry \& Padovani (1995) infer a $p$ value of $\approx 3$ based on the observations of superluminal motion within our own galaxy by Mirabel \& Rodriguez (1994). We get,

$$
\begin{aligned}
\beta \cos \theta= & \left(1-\frac{(2 b)^{2 / 3}}{\sqrt{R_{\mathrm{c}}}\left(-2 \sqrt{R_{\mathrm{c}}}+\sqrt{2 b+4 R_{\mathrm{c}}}\right)^{1 / 3}}\right. \\
& \left.+\frac{(2 b)^{1 / 3}\left(-2 \sqrt{R_{\mathrm{c}}}+\sqrt{2 b+4 R_{\mathrm{c}}}\right)^{1 / 3}}{\sqrt{R_{\mathrm{c}}}}\right)^{1 / 2}
\end{aligned}
$$

where $b=\frac{R_{\mathrm{c}}^{\mathrm{int}}}{\gamma^{p}}$.

\section{Appendix B: The models and the fitting procedure}

We describe here the model-fitting procedure and the use of the Akaike's information criterion (AIC) to evaluate the models. We considered several simple models to compare the behaviour of the optical nuclei in the FRI and FRII populations. For the "Jet-only" model, the entire nuclear optical luminosity is ascribed to synchrotron emission from a jet which is relativistically beamed. For the "Jet+Disk" model, the nuclear optical luminosity is modelled as a combination of a beamed synchrotron jet and a thin optically thick disk. For the "Jet+Disk+Torus" model, Eq. (A.1) is used in toto. In the "Jet+Torus" model the entire nuclear optical luminosity is due to the beamed jet, modified by an obscuring torus.

Using the $\gamma$ values derived as in Eq. (A.3) from the minimum and maximum values of $R_{\mathrm{c}}$ in our data set for each 
of the FRI and FRII populations and Eqs. (A.1) and (A.5) for $L_{\mathrm{o}}$ and $R_{\mathrm{c}}$, we made a non-linear least squares fit to the data for both the FR populations separately. We used the Levenberg-Marquardt (LM) algorithm as implemented in the IDL package (the LMFIT routine). This routine gives the best-fit values of the free parameters, their standard deviations and the $\chi^{2}$ goodness-of-fit. For the model-fitting we assumed the errors in the nuclear optical luminosity to be $50 \%$ (e.g., Verdoes Kleijn et al. 2002).

The Akaike's information criterion is used to compare different model-fits and is defined as

$\mathrm{AIC}=-2 \ln (L)+2 k$

(Burnham \& Anderson 2002),

where $\ln (L)$ is the $\log$ likelihood function and is given by

$\ln (L)=-(n / 2)\{\ln (2 \pi)+\ln (\mathrm{SEE} / n)+1\}$

where " $n$ " is the number of data-points, "SEE" is the Standard error of estimate and " $k$ " is the number of parameters to be fitted. Smaller AIC values indicate a better fit. We note that the goodness-of-fit criterion AIC behaves in an inverse fashion to the $\chi^{2}$ probability $Q$ (Press et al. 1992).

For the "Jet-only" model, $L_{\text {jet }}^{\text {int }}$ is the only free parameter and the LM algorithm yields its best fit value. For the "Jet+Disk" model, both $L_{\text {jet }}^{\text {int }}$ and $L_{\text {disk }}$ are free parameters. The output of the algorithm turned out to be independent of the input seed values of these free parameters. The "Jet+Disk+Torus" and the "Jet+Torus" models have two additional parameters of the torus, viz., $A_{V_{0}}$ and $\theta_{\mathrm{c}}$. However, apart from the quality of the data, the fact that there is more than one "local minimum" for $\theta_{c}$ (discussed in Sect. 3.5.1) did not allow a robust estimation of all the parameters simultaneously. (The estimated values for $\theta_{\mathrm{c}}$ strongly depended on the input seed value.) We therefore adopted the procedure of manually varying the extinction $A_{V_{0}}$ and torus half-opening angle $\theta_{\mathrm{c}}$ and using the resulting AIC for each pair of (fixed) $A_{V_{0}}$ and $\theta_{\mathrm{c}}$ to infer the best fit.

Considering first the most general "Jet+Disk+Torus" model to the fit the data, we chose a range of values for the torus parameters, $A_{V_{0}}$ and $\theta_{\mathrm{c}}$, and estimated the best fit $L_{\text {jet }}^{\text {int }}$ and $L_{\text {disk }}$ using the LM algorithm. The results are tabulated in Table 2 for representative values, $A_{V_{0}}=3$ and $\theta_{\mathrm{c}}=45^{\circ}$ (e.g., Barthel 1989). The resulting value of $L_{\text {disk }}$ turned out to be insignificant in each case (see Table 2 and Sect. 3.5.1). We therefore further considered only the "Jet+Torus" model which seemed more applicable to the data. For a given value of $A_{V_{0}}$ we varied the $\theta_{\mathrm{c}}$ from $0^{\circ}$ through $90^{\circ}$ and tabulated the resultant AIC, which are plotted in Fig. 6.

As a next step, we fixed the torus half-opening angle $\theta_{\mathrm{c}}$ to the value which had resulted in the minimum AIC (Fig. 6) which is approximately the same for different values of $A_{V_{0}}$ for each FR population, and let the $A_{V_{0}}$ be the free parameter to be best-fitted by the LM algorithm. We found that the resul$\operatorname{tant} A_{V_{0}}$ for the FRII objects was independent of the seed value whereas it depended on the initial value for the FRI objects. In this manner we estimated the "Best fit" "Jet+Torus" model with values of $A_{V_{0}}$ and $\theta_{\mathrm{c}}$ which gave the lowest AIC value.

\section{References}

Akaike, H. 1974, IEEE Trans. Automatic Control, 19, 716

Baker, J. C., Hunstead, R. W., Kapahi, V. K., \& Subrahmanya, C. R. 1994, in The First Stromlo Symp.: The Physics of Active Galaxies, ed. G. V. Bicknell, M. A. Dopita, \& P. J. Quinn, 195

Barthel, P. D. 1989, ApJ, 336, 606

Baum, S. A., Zirbel, E. L., \& O’Dea, C. P. 1995, ApJ, 451, 88

Becker, R. H., White, R. L., \& Edwards, A. L. 1991, ApJS, 75, 1

Blandford, R. D., \& Konigl, A. 1979, ApJ, 232, 34

Bridle, A. H., \& Perley, R. A. 1984, ARA\&A, 22, 319

Burnham, K. P., \& Anderson, D. R. 2002, Model selection and multimodel inference: a practical information-theoretic approach (New York, NY: Springer-Verlag)

Capetti, A., \& Celotti, A. 1999, MNRAS, 304, 434

Capetti, A., Fanti, R., \& Parma, P. 1995, A\&A, 300, 643

Capetti, A., Celotti, A., Chiaberge, M., et al. 2002, A\&A, 383, 104

Cassaro, P., Stanghellini, C., Bondi, M., et al. 1999, A\&AS, 139, 601

Chiaberge, M., Capetti, A., \& Celotti, A. 1999, A\&A, 349, 77

Chiaberge, M., Capetti, A., \& Celotti, A. 2000, A\&A, 355, 873

Chiaberge, M., Capetti, A., \& Celotti, A. 2002, A\&A, 394, 791

de Koff, S., Best, P., Baum, S. A., et al. 2000, ApJS, 129, 33

Falomo, R., Scarpa, R., \& Bersanelli, M. 1994, ApJS, 93, 125

Fanaroff, B. L., \& Riley, J. M. 1974, MNRAS, 167, 31P

Fomalont, E. B., \& Bridle, A. H. 1978, AJ, 83, 725

Giovannini, G., Feretti, L., Gregorini, L., \& Parma, P. 1988, A\&A, 199,73

Gregory, P. C., \& Condon, J. J. 1991, ApJS, 75, 1011

Hardcastle, M. J., Alexander, P., Pooley, G. G., \& Riley, J. M. 1996, MNRAS, 278, 273

Hardcastle, M. J., \& Worrall, D. M. 1999, MNRAS, 309, 969

Hardcastle, M. J., \& Worrall, D. M. 2000, MNRAS, 314, 359

Impey, C. D., \& Tapia, S. 1990, ApJ, 354, 124

Jannuzi, B. T., Yanny, B., \& Impey, C. 1997, ApJ, 491, 146

Jenkins, C. R. 1982, MNRAS, 200, 705

Kapahi, V. K., \& Saikia, D. J. 1982, J. A\&A, 3, 465

Kapahi, V. K., \& Shastri, P. 1987, MNRAS, 224, 17P

Kollgaard, R. I., Wardle, J. F. C., Roberts, D. H., \& Gabuzda, D. C. 1992, AJ, 104, 1687

Kotilainen, J. K., Falomo, R., \& Scarpa, R. 1998, A\&A, 336, 479

Kuehr, H., Nauber, U., \& Pauliny-Toth, I. I. K. 1979, A Catalogue of radio sources (Bonn: Max-Planck-Institut (MPI) für Radioastronomie)

Kuehr, H., Pauliny-Toth, I. I. K., Witzel, A., \& Schmidt, J. 1981, AJ, 86,854

Laing, R. A., Parma, P., de Ruiter, H. R., \& Fanti, R. 1999, MNRAS, 306,513

Laurent-Muehleisen, S. A., Kollgaard, R. I., Moellenbrock, G. A., \& Feigelson, E. D. 1993, AJ, 106, 875

Laurent-Muehleisen, S. A., Kollgaard, R. I., Ryan, P. J., et al. 1997, A\&AS, 122, 235

Ledlow, M. J., \& Owen, F. N. 1996, AJ, 112, 9

Meier, D. L. 1999, ApJ, 522, 753

Mirabel, I. F., \& Rodriguez, L. F. 1994, Nature, 371, 46

Morris, S. L., Stocke, J. T., Gioia, I. M., et al. 1991, ApJ, 380, 49

O'Dea, C. P., Barvainis, R., \& Challis, P. M. 1988, AJ, 96, 435

Orr, M. J. L., \& Browne, I. W. A. 1982, MNRAS, 200, 1067

Padovani, P., \& Giommi, P. 1995, MNRAS, 277, 1477

Perlman, E. S., \& Stocke, J. T. 1993, ApJ, 406, 430

Perlman, E. S., Sparks, W. B., Radomski, J., et al. 2001, ApJ, 561, L51

Pica, A. J., Smith, A. G., Webb, J. R., et al. 1988, AJ, 96, 1215

Press, W. H., Flannery, B. P., Teukolsky, S. A., \& Vetterling, W. T. 1992, Numerical Recipes in Fortran (Cambridge University Press) 
Prestage, R. M., \& Peacock, J. A. 1988, MNRAS, 230, 131

Rector, T. A., \& Stocke, J. T. 2001, AJ, 122, 565

Simpson, C. 1996, Vistas Astron., 40, 57

Slee, O. B., Siegman, B. C., \& Perley, R. A. 1989, Aust. J. Phys., 42, 633

Smith, E. P., \& Heckman, T. M. 1990, ApJ, 348, 38

Spangler, S. R., \& Sakurai, T. 1985, ApJ, 297, 84

Urry, C. M., \& Padovani, P. 1995, PASP, 107, 803

Verdoes Kleijn, G. A., Baum, S. A., de Zeeuw, P. T., \& O'Dea, C. P. 1999, AJ, 118, 2592

Verdoes Kleijn, G. A., Baum, S. A., de Zeeuw, P. T., \& O’Dea, C. P. Zirbel, E. L., \& Baum, S. A. 1995, ApJ, 448, 521
Vermeulen, R. C., \& Cohen, M. H. 1994, ApJ, 430, 467

Véron-Cetty, M. P., \& Véron, P. 1998, VizieR Online Data Catalog, 7207,0

Waggett, P. C., Warner, P. J., \& Baldwin, J. E. 1977, MNRAS, 181, 465

Webb, J. R., Smith, A. G., Leacock, R. J., et al. 1988, AJ, 95, 374

Wills, B. J., Wills, D., Breger, M., Antonucci, R. R. J., \& Barvainis, R. 1992, ApJ, 398, 454

Xu, C., Baum, S. A., O’Dea, C. P., Wrobel, J. M., \& Condon, J. J. 2000, AJ, 120, 2950

2002, AJ, 123, 1334 


\section{Online Material}


P. Kharb and P. Shastri: Optical nuclei and the F-R Divide, Online Material p 2

\section{Appendix C: Data on sample objects}

Table C.1. The FRI radio galaxies.

\begin{tabular}{|c|c|c|c|c|c|c|c|c|c|}
\hline $\begin{array}{l}\text { IAU } \\
\text { name }\end{array}$ & $\begin{array}{c}\text { Alternate } \\
\text { name }\end{array}$ & $\begin{array}{c}\text { Redshift } \\
z \\
\end{array}$ & $b / a$ & $\begin{array}{r}\log L_{\text {ext }} \\
\mathrm{W} \mathrm{Hz}^{-1}\end{array}$ & $\begin{array}{c}S_{\mathrm{c}}(5 \mathrm{GHz}) \\
\mathrm{mJy}\end{array}$ & Ref. & $\log R_{\mathrm{c}}$ & $\begin{array}{c}\log L_{\mathrm{o}} \\
\mathrm{W} \mathrm{Hz} \\
\end{array}$ & Ref \\
\hline $0036+030$ & NGC 193 & 0.0144 & $0.18^{l}$ & 22.60 & 40.0 & 6,15 & -1.31 & 18.35 & 5 \\
\hline $0053+261$ & 3C 28 & 0.1952 & $\ldots$ & 25.42 & $<0.2$ & 1,3 & $<-2.92$ & $<19.48$ & 1 \\
\hline 0055-016 & 3C 29 & 0.0448 & $\ldots$ & 25.29 & 93.0 & 1,3 & -1.39 & 19.43 & 1 \\
\hline $0055+265$ & 4C 26.03 & 0.0472 & $\ldots$ & 24.61 & 9.0 & 2 & -1.93 & $<18.54$ & 2 \\
\hline $0055+300$ & NGC 315 & 0.0167 & $0.23^{d}$ & 24.01 & 617.6 & 2 & -0.39 & 19.19 & 5 \\
\hline $0104+321$ & 3C 31 & 0.0169 & $0.77^{d}$ & 24.40 & 92.0 & 1,3 & -1.34 & 19.08 & 5 \\
\hline $0120+329$ & NGC 507 & 0.0164 & . & 22.29 & 1.5 & 2 & -1.30 & $<17.64$ & 2 \\
\hline 0123-016 & $3 \mathrm{C} 40$ & 0.0180 & $0.91^{d}$ & 22.17 & 67.8 & 10 & -1.64 & $<18.63$ & 5 \\
\hline $0153+053$ & NGC 741 & 0.0185 & $\ldots$ & 22.75 & 6.0 & 11 & -1.65 & $<18.35$ & 5 \\
\hline $0220+427$ & $3 \mathrm{C} 66 \mathrm{~B}$ & 0.0215 & $0.98^{d}$ & 24.86 & 182.0 & 1,3 & -1.29 & 19.62 & 5 \\
\hline $0305+039$ & $3 \mathrm{C} 78$ & 0.0288 & $\ldots$ & 24.96 & 964.0 & 1,3 & -0.42 & 20.66 & 1 \\
\hline $0318+415$ & $3 C 83.1$ & 0.0251 & $0.09^{d}$ & 24.98 & 21.0 & 1,3 & -2.22 & 18.31 & 1 \\
\hline $0316+413$ & 3C 84 & 0.0176 & $\ldots$ & 24.73 & 42370.0 & 1,3 & 1.02 & 21.03 & 1 \\
\hline 0331-013 & $3 \mathrm{C} 89$ & 0.1386 & & 25.80 & 49.0 & 1,3 & -1.21 & $<18.97$ & 1 \\
\hline $0705+486$ & NGC 2329 & 0.0193 & $0.68^{d}$ & 23.02 & 69.0 & 11 & -0.44 & 20.04 & 5 \\
\hline $0755+379$ & 3C 189 & 0.0413 & $\ldots$ & 24.43 & 228.8 & 2 & -0.46 & 20.18 & 2 \\
\hline $0924+301$ & $\ldots$ & 0.0266 & $\ldots$ & 23.52 & 0.4 & 2 & $<-2.64$ & $<17.98$ & 2 \\
\hline $0928+678$ & NGC 2892 & 0.0225 & $\ldots$ & 22.82 & 30.0 & 12 & -0.52 & 19.15 & 5 \\
\hline $1142+198$ & 3C 264 & 0.0206 & $0.99^{d}$ & 24.57 & 200.0 & 1,3 & -1.00 & 20.15 & 5 \\
\hline $1205+255$ & UGC 7115 & 0.0226 & $\ldots$ & 22.58 & 44.0 & 11 & -0.51 & 19.48 & 5 \\
\hline $1216+061$ & $3 \mathrm{C} 270$ & 0.0074 & $0.46^{d}$ & 24.31 & 308.0 & 1,3 & -1.44 & 17.56 & 5 \\
\hline $1220+587$ & NGC 4335 & 0.0154 & $0.41^{d}$ & 22.64 & 15.0 & 6 & -0.44 & $<19.01$ & 5 \\
\hline $1222+131$ & 3C 272.1 & 0.0037 & $0.15^{l}$ & 23.22 & 180.0 & 1,3 & -1.18 & 18.17 & 5 \\
\hline $1228+126$ & $3 \mathrm{C} 274$ & 0.0037 & $\ldots$ & 24.63 & 4000.0 & 1,3 & -1.24 & 19.18 & 5 \\
\hline $1257+282$ & NGC 4874 & 0.0239 & $\ldots$ & 23.07 & 1.2 & 2 & -1.87 & $<17.49$ & 2 \\
\hline $1322+366$ & NGC 5141 & 0.0173 & $0.25^{l}$ & 23.63 & 78.7 & 10 & -0.61 & $<18.69$ & 5 \\
\hline $1336+391$ & $3 \mathrm{C} 288$ & 0.2460 & $\ldots$ & 26.42 & 30.0 & 1,3 & -1.56 & 20.03 & 1 \\
\hline $1346+268$ & 4C 26.42 & 0.0633 & $\ldots$ & 24.52 & 59.3 & 2 & -0.78 & 19.58 & 2 \\
\hline $1407+177$ & NGC 5490 & 0.0162 & $0.35^{l}$ & 23.24 & 37.8 & 10 & -1.28 & $<17.48$ & 5 \\
\hline $1414+110$ & 3C 296 & 0.0237 & $0.29^{d}$ & 24.61 & 77.0 & 1,3 & -1.33 & 18.64 & 1 \\
\hline $1422+268$ & $\ldots$ & 0.0370 & $\ldots$ & 23.99 & 21.1 & 2 & -1.15 & $<19.41$ & 2 \\
\hline $1430+251$ & 4C 25.46 & 0.0813 & $\ldots$ & 24.20 & 1.2 & 2 & $<-1.93$ & $<19.61$ & 2 \\
\hline $1450+281$ & $\ldots$ & 0.1265 & $\ldots$ & 24.48 & 6.7 & 2 & -1.12 & $<19.77$ & 2 \\
\hline $1502+261$ & 3C 310 & 0.0540 & $\ldots$ & 25.19 & 80.0 & 1,3 & -1.19 & 19.38 & 1 \\
\hline $1510+709$ & $3 C 314.1$ & 0.1197 & $\ldots$ & 25.33 & $<1.0$ & 1,3 & $<-2.55$ & $<19.52$ & 1 \\
\hline $1514+072$ & $3 C 317$ & 0.0342 & $\ldots$ & 24.43 & 391.0 & 1,3 & -0.13 & 19.49 & 1 \\
\hline $1521+288$ & 4C 28.39 & 0.0825 & $\ldots$ & 24.53 & 55.8 & 2 & -0.60 & 20.20 & 2 \\
\hline $1527+308$ & $\ldots$ & 0.1143 & $\ldots$ & 24.03 & 4.0 & 2 & -0.97 & $<19.89$ & 2 \\
\hline $1553+245$ & $\ldots$ & 0.0426 & $\ldots$ & 23.43 & 57.9 & 2 & -0.03 & 19.84 & 2 \\
\hline $1610+296$ & NGC 6086 & 0.0313 & $\ldots$ & 22.92 & 1.1 & 2 & $<-1.52$ & $<18.38$ & 2 \\
\hline $1613+275$ & $\ldots$ & 0.0647 & $\ldots$ & 24.01 & 10.6 & 2 & -1.00 & $<19.24$ & 2 \\
\hline $1626+396$ & 3C 338 & 0.0303 & $\ldots$ & 24.19 & 105.0 & 1,3 & -0.57 & 19.33 & 1 \\
\hline $1637+826$ & NGC 6251 & 0.024 & $\ldots$ & 23.82 & 720.0 & 13,4 & 0.44 & 20.19 & 7 \\
\hline $1641+173$ & 3C 346 & 0.1620 & $\ldots$ & 26.20 & 220.0 & 1,3 & -0.82 & 21.17 & 1 \\
\hline $1648+050$ & 3C 348 & 0.1540 & $\ldots$ & 27.12 & 10.0 & 1,3 & -3.13 & 19.67 & 1 \\
\hline $1827+323$ & $\ldots$ & 0.0659 & $\ldots$ & 24.04 & 20.8 & 2 & -0.71 & $<19.38$ & 2 \\
\hline $2045+068$ & $3 \mathrm{C} 424$ & 0.1270 & $\ldots$ & 25.67 & 18.0 & 1,3 & -1.58 & $<19.77$ & 1 \\
\hline $2116+262$ & NGC 7052 & 0.0164 & $0.30^{d}$ & 22.97 & 47.0 & 9,14 & -0.22 & 18.26 & 8 \\
\hline $2153+377$ & 3C 438 & 0.2900 & $\ldots$ & 26.77 & 17.0 & 1,3 & -2.02 & $<19.94$ & 1 \\
\hline $2212+135$ & $3 \mathrm{C} 442$ & 0.0262 & $\ldots$ & 24.39 & 2.0 & 1,3 & -2.61 & 18.16 & 1 \\
\hline $2229+391$ & 3C 449 & 0.0181 & $0.50^{d}$ & 24.29 & 37.0 & 1,3 & -1.56 & 19.13 & 1 \\
\hline $2236+350$ & UGC 12127 & 0.0277 & $\ldots$ & 23.46 & 7.1 & 2 & -1.34 & 18.55 & 2 \\
\hline $2318+079$ & NGC 7626 & 0.0113 & $0.17^{l}$ & 22.39 & 15.6 & 10 & -1.47 & $<17.91$ & 5 \\
\hline $2335+267$ & $3 \mathrm{C} 465$ & 0.0301 & $0.69^{l}$ & 25.00 & 270.0 & 1,3 & -0.97 & 19.60 & 1 \\
\hline
\end{tabular}

Superscripts " $d$ " and "l" for $b / a$ stand for extended dust disks and lanes, respectively. References: (1): Chiaberge et al. (1999) ( $F 702 W$ filter); For the 7 sources which were common between the 3CR, B2 and the UGC samples we used the $F 555 W$ flux densities from Verdoes Kleijn et al. (2002); (2): Capetti et al. (2002) (1.4 GHz, F814W filter); (3): Kuehr et al. (1979) (5 GHz); (4): Kuehr et al. (1981) (5 GHz); (5): Verdoes Kleijn et al. (2002) ( $F 555 W$ filter); (6): Xu et al. (2000) (1.4 GHz); (7): Hardcastle \& Worrall (1999) (F702W filter); (8): (Capetti \& Celotti 1999) ( $F 814 W$ filter); (9): Giovannini et al. (1988) (5 GHz); (10): Bridle \& Perley (1984) (core at $5 \mathrm{GHz}$, total flux density at $1.4 \mathrm{GHz}$ ); (11): Laurent-Muehleisen et al. (1997) (5 GHz); (12): Jenkins (1982) (5 GHz); (13): Waggett et al. (1977) (2.7 GHz); (14): Gregory \& Condon (1991) (5 GHz); (15): Becker et al. (1991) (5 GHz). 
P. Kharb and P. Shastri: Optical nuclei and the F-R Divide, Online Material p 3

Table C.2. The FRII radio galaxies.

\begin{tabular}{|c|c|c|c|c|c|c|c|c|c|}
\hline $\begin{array}{l}\text { IAU } \\
\text { name }\end{array}$ & $\begin{array}{l}\text { Alternate } \\
\text { name }\end{array}$ & $\begin{array}{c}\text { Redshift } \\
z\end{array}$ & $b / a$ & $\begin{array}{l}\log L_{\mathrm{ext}} \\
\mathrm{W} \mathrm{Hz}^{-1}\end{array}$ & $\begin{array}{c}S_{\mathrm{c}}(5 \mathrm{GHz}) \\
\mathrm{mJy}\end{array}$ & Ref. & $\log R_{\mathrm{c}}$ & $\begin{array}{c}\log L_{\mathrm{o}} \\
\mathrm{W} \mathrm{Hz}\end{array}$ & Ref. \\
\hline 0034-014 & $3 \mathrm{C} 15$ & 0.073 & $\ldots$ & 25.46 & 372.8 & 1,3 & -0.52 & $<19.74$ & 1 \\
\hline $0035-024$ & $3 \mathrm{C} 17^{\star}$ & 0.220 & $\ldots$ & 26.63 & 727.9 & 1,3 & -0.48 & 21.64 & 1 \\
\hline $0038+097$ & 3C 18 & 0.188 & $\ldots$ & 26.41 & 118.2 & 1,3 & -1.18 & 21.36 & 1 \\
\hline $0106+729$ & $3 \mathrm{C} 33.1^{\star}$ & 0.181 & $\ldots$ & 26.11 & 19.7 & 1,3 & -1.69 & 20.94 & 1 \\
\hline $0109+492$ & 3C 35 & 0.067 & $\ldots$ & 25.06 & 23.7 & 1,3 & -1.40 & $<19.31$ & 1 \\
\hline 0218-021 & $3 \mathrm{C} 63$ & 0.175 & $\ldots$ & 26.10 & 18.3 & 1,3 & -1.74 & 21.04 & 1 \\
\hline $0307+169$ & $3 \mathrm{C} 79$ & 0.256 & $\ldots$ & 26.62 & 14.7 & 1,3 & -2.03 & 21.02 & 1 \\
\hline $0325+023$ & $3 C 88$ & 0.030 & $\ldots$ & 24.86 & 197.2 & 1,3 & -0.97 & 19.46 & 1 \\
\hline $0356+102$ & 3C 98 & 0.030 & $\ldots$ & 25.25 & 11.1 & 1,3 & -2.61 & $<18.53$ & 1 \\
\hline $0415+379$ & $3 \mathrm{C} 111^{\star}$ & 0.049 & $\ldots$ & 25.86 & 1155.3 & 1,4 & -0.77 & 20.99 & 1 \\
\hline $0433+295$ & $3 \mathrm{C} 123$ & 0.218 & $\ldots$ & 27.55 & 85.0 & 1,3 & -2.33 & $<19.16$ & 1 \\
\hline $0453+227$ & $3 \mathrm{C} 132$ & 0.214 & $\ldots$ & 26.35 & 33.5 & 1,3 & -1.56 & $<19.55$ & 1 \\
\hline $0459+252$ & $3 \mathrm{C} 133$ & 0.277 & $\ldots$ & 26.85 & 170.8 & 1,3 & -1.14 & 20.68 & 1 \\
\hline $0511+008$ & $3 C 135$ & 0.127 & $\ldots$ & 25.88 & 5.5 & 1,3 & -2.31 & 20.15 & 1 \\
\hline $0605+480$ & $3 \mathrm{C} 153$ & 0.277 & $\ldots$ & 26.68 & 0.4 & 1,3 & -3.56 & $<19.34$ & 1 \\
\hline $0640+233$ & $3 \mathrm{C} 165$ & 0.296 & $\ldots$ & 26.48 & 8.7 & 1,3 & -2.01 & 19.95 & 1 \\
\hline $0642+214$ & 3C 166 & 0.245 & $\ldots$ & 26.15 & 553.6 & 1,3 & -0.03 & 20.71 & 1 \\
\hline $0651+542$ & $3 \mathrm{C} 171$ & 0.238 & $\ldots$ & 26.49 & 2.5 & 1,3 & -2.74 & 19.20 & 1 \\
\hline $0734+805$ & $3 \mathrm{C} 184.1$ & 0.118 & $\ldots$ & 25.87 & 7.5 & 1,3 & -2.23 & 20.96 & 1 \\
\hline $0802+243$ & $3 \mathrm{C} 192$ & 0.060 & $\ldots$ & 25.56 & 8.5 & 1,3 & -2.44 & $<19.94$ & 1 \\
\hline $0818+472$ & $3 \mathrm{C} 197.1$ & 0.131 & $\ldots$ & 25.82 & 6.8 & 1,3 & -2.13 & 20.88 & 1 \\
\hline $0819+061$ & 3C 198 & 0.082 & $\ldots$ & 25.14 & $<1.5$ & 5,4 & $<-2.50$ & 20.85 & 1 \\
\hline $0917+458$ & $3 \mathrm{C} 219^{\star}$ & 0.174 & $\ldots$ & 26.49 & 68.7 & 1,3 & -1.56 & 21.39 & 1 \\
\hline $0936+361$ & $3 C 223$ & 0.137 & $\ldots$ & 26.04 & 11.7 & 1,3 & -2.07 & $<20.05$ & 1 \\
\hline $0938+399$ & $3 \mathrm{C} 223.1$ & 0.108 & $\ldots$ & 25.65 & 8.7 & 1,3 & -2.02 & $<19.98$ & 1 \\
\hline $0945+076$ & $3 \mathrm{C} 227^{\star}$ & 0.086 & $\ldots$ & 25.94 & 23.5 & 1,3 & -2.07 & 21.66 & 1 \\
\hline $0958+290$ & $3 \mathrm{C} 234^{\star}$ & 0.185 & $\ldots$ & 26.34 & 133.6 & 1,3 & -1.07 & 21.85 & 1 \\
\hline $1003+351$ & $3 \mathrm{C} 236$ & 0.099 & $\ldots$ & 25.70 & 191.5 & 1,3 & -0.80 & $<19.84$ & 1 \\
\hline $1205+341$ & $\ldots$ & 0.0788 & $\ldots$ & 24.46 & 12.5 & 2 & -1.21 & $<19.81$ & 2 \\
\hline $1251+278$ & $3 C 277.3$ & 0.0857 & $\ldots$ & 25.37 & 12.4 & 2 & -2.05 & 19.49 & 2 \\
\hline $1319+428$ & $3 \mathrm{C} 285$ & 0.079 & $\ldots$ & 25.32 & 7.8 & 1,3 & -2.00 & 18.47 & 1 \\
\hline $1330+022$ & $3 C 287.1^{\star}$ & 0.216 & $\ldots$ & 26.34 & 443.8 & 1,3 & -0.42 & 21.43 & 1 \\
\hline $1420+198$ & 3C 300 & 0.270 & $\ldots$ & 26.58 & 10.1 & 1,3 & -2.12 & 20.47 & 1 \\
\hline $1441+522$ & $3 C 303^{\star}$ & 0.141 & $\ldots$ & 25.83 & 187.6 & 1,3 & -0.64 & 21.44 & 1 \\
\hline $1519+078$ & $3 \mathrm{C} 318.1$ & 0.046 & $\ldots$ & 24.49 & 3.0 & 6,3 & -2.05 & $<18.52$ & 1 \\
\hline $1522+546$ & 3C 319 & 0.192 & $\ldots$ & 26.04 & 1.4 & 1,3 & -2.72 & $<20.14$ & 1 \\
\hline $1545+210$ & $3 \mathrm{C} 323.1^{\star}$ & 0.264 & $\ldots$ & 26.44 & 43.8 & 1,3 & -1.36 & 22.83 & 1 \\
\hline $1549+202$ & 3C 326 & 0.089 & $0.24^{d}$ & 25.19 & 15.9 & 1,3 & -1.46 & $<19.91$ & 1 \\
\hline $1559+021$ & $3 \mathrm{C} 327$ & 0.104 & $\ldots$ & 26.18 & 40.8 & 1,3 & -1.91 & $<19.35$ & 1 \\
\hline $1615+325$ & $3 \mathrm{C} 332^{\star}$ & 0.152 & $\ldots$ & 25.93 & 11.5 & 1,3 & -1.89 & 21.56 & 1 \\
\hline $1658+471$ & $3 \mathrm{C} 349$ & 0.205 & $\ldots$ & 26.33 & 21.9 & 1,3 & -1.76 & 20.88 & 1 \\
\hline $1717-009$ & $3 C 353$ & 0.030 & $\ldots$ & 25.94 & 216.2 & 1,3 & -2.01 & $<18.40$ & 1 \\
\hline $1726+318$ & 3C 357 & 0.167 & $\ldots$ & 26.10 & 6.5 & 1,3 & -2.23 & $<19.66$ & 1 \\
\hline $1825+743$ & $3 \mathrm{C} 379.1$ & 0.256 & $\ldots$ & 26.32 & 3.9 & 7,3 & -2.32 & $<19.91$ & 1 \\
\hline $1832+474$ & $3 \mathrm{C} 381$ & 0.161 & $\ldots$ & 26.18 & 6.9 & 1,3 & -2.31 & $<20.28$ & 1 \\
\hline $1833+326$ & $3 \mathrm{C} 382^{\star}$ & 0.058 & $\ldots$ & 25.47 & 228.1 & 1,4 & -0.95 & 22.56 & 1 \\
\hline $1842+455$ & 3C 388 & 0.091 & $\ldots$ & 25.80 & 76.5 & 1,3 & -1.37 & 20.07 & 1 \\
\hline $1845+797$ & 3 C $390.3^{\star}$ & 0.056 & $\ldots$ & 25.74 & 434.7 & 1,3 & -0.97 & 21.88 & 1 \\
\hline $1939+605$ & 3C 401 & 0.201 & $\ldots$ & 26.41 & 47.5 & 1,3 & -1.52 & 20.35 & 1 \\
\hline $1940+505$ & $3 \mathrm{C} 402$ & 0.025 & $\ldots$ & 24.39 & 48.1 & 1,3 & -1.27 & 19.46 & 1 \\
\hline $1949+023$ & $3 \mathrm{C} 403$ & 0.059 & $\ldots$ & 25.54 & 12.1 & 1,3 & -2.28 & 19.49 & 1 \\
\hline $2221-023$ & $3 \mathrm{C} 445^{\star}$ & 0.057 & $\ldots$ & 25.40 & 382.8 & 1,3 & -0.66 & 22.11 & 1 \\
\hline $2243+394$ & $3 \mathrm{C} 452$ & 0.081 & $0.27^{l}$ & 25.96 & 152.3 & 1,3 & -1.33 & $<19.72$ & 1 \\
\hline $2309+090$ & $3 C 456$ & 0.233 & $\ldots$ & 26.24 & 27.8 & 1,3 & -1.45 & 21.21 & 1 \\
\hline $2318+235$ & $3 \mathrm{C} 460$ & 0.268 & $\ldots$ & 26.02 & 21.4 & 1,4 & -1.24 & 20.29 & 1 \\
\hline
\end{tabular}

Superscripts " $d$ " and " $l$ " for $b / a$ stand for extended dust disks and lanes, respectively. There are only two FRIIs for which $b / a$ for extended dust features are available (de Koff et al. 2000). We do not include the FRIIs in our analysis of the extended dusty disks. Sources with a star are BLRGs. References: (1): Chiaberge et al. (2002) (5 GHz, F702W filter, except 3C 192 observed with F555W); (2): Capetti et al. (2002) (1.4 GHz); (3): Kuehr et al. (1979) (5 GHz); (4): Véron-Cetty \& Véron (1998) (5 GHz); (5): Fomalont \& Bridle (1978) (5 GHz); (6): Slee et al. (1989) (1.5 GHz); (7): Spangler \& Sakurai (1985) (1.4 GHz). 
P. Kharb and P. Shastri: Optical nuclei and the F-R Divide, Online Material $p 4$

Table C.3. The BL Lac objects.

\begin{tabular}{|c|c|c|c|c|c|c|c|c|c|}
\hline $\begin{array}{l}\text { IAU } \\
\text { name }\end{array}$ & $\begin{array}{c}\text { Alternate } \\
\text { name }\end{array}$ & $\begin{array}{c}\text { Redshift } \\
z\end{array}$ & $m_{v}$ & $\overline{\text { Ref. }}$ & $\begin{array}{l}\log L_{\text {ext }} \\
\mathrm{W} \mathrm{Hz}^{-1}\end{array}$ & $\begin{array}{c}S_{\mathrm{c}}(5 \mathrm{GHz}) \\
\mathrm{mJy}\end{array}$ & Ref. & $\overline{l o g} R_{\mathrm{c}}$ & $\begin{array}{c}\log L_{\mathrm{o}} \\
\mathrm{W} \mathrm{Hz}{ }^{-1}\end{array}$ \\
\hline $0158+003$ & $\ldots$ & 0.299 & 17.96 & 1 & 24.36 & 8.38 & 2 & 0.60 & 22.61 \\
\hline 0219-164 & $\ldots$ & 0.698 & 17.0 & 9 & 25.50 & 358.0 & 5 & 0.84 & 23.78 \\
\hline $0219+428$ & $3 \mathrm{C} 66 \mathrm{~A}$ & 0.444 & 15.08 & 6 & 27.32 & 814.0 & $2^{a}$ & 0.14 & 24.13 \\
\hline $0257+344$ & $\ldots$ & 0.247 & 18.53 & 1 & 23.22 & 11.78 & 2 & 1.69 & 22.21 \\
\hline $0317+185$ & $\ldots$ & 0.190 & 18.12 & 1 & 23.50 & 9.85 & 2 & 1.08 & 22.13 \\
\hline $0323+022$ & $\ldots$ & 0.147 & 16.98 & 6 & 23.20 & 55.0 & 5 & 1.21 & 22.36 \\
\hline $0414+009$ & $\ldots$ & 0.287 & 17.11 & 6 & 24.50 & 67.0 & 5 & 0.54 & 22.91 \\
\hline $0454+844$ & $\cdots$ & $1.34^{\star}$ & 17.3 & 9 & 24.21 & 1400.0 & 4 & $>3.37^{\dagger}$ & 24.29 \\
\hline $0521-365$ & $\ldots$ & 0.055 & 14.62 & 9 & 26.12 & 3124.0 & $2^{a}$ & -0.14 & 22.43 \\
\hline $0548-322$ & $\ldots$ & 0.069 & 16.05 & 8 & 24.67 & 80.0 & $2^{a}$ & -0.08 & 22.06 \\
\hline $0607+711$ & $\ldots$ & 0.267 & 19.60 & 1 & 24.79 & 14.08 & 2 & 0.29 & 21.85 \\
\hline $0706+592$ & $\ldots$ & 0.124 & 18.40 & 9 & 24.20 & 65.0 & 5 & 0.17 & 21.64 \\
\hline $0735+178$ & $\ldots$ & $>0.424$ & 15.40 & 9 & 23.82 & 1990.0 & 4 & $>3.2$ & 23.96 \\
\hline $0737+746$ & $\ldots$ & 0.315 & 16.89 & 1 & 23.85 & 24.47 & 2 & 1.64 & 23.09 \\
\hline $0851+202$ & OJ287 & 0.306 & 13.81 & 7 & 24.21 & 2217.0 & $2^{a}$ & 3.23 & 24.29 \\
\hline $1101-232$ & $\ldots$ & 0.186 & 17.01 & 8 & 24.40 & 49.0 & 5 & 0.41 & 22.56 \\
\hline $1101+384$ & Mrk 421 & 0.030 & 13.22 & 6 & 23.85 & 520.0 & $2^{a}$ & 0.81 & 22.46 \\
\hline $1133+704$ & Mrk 180 & 0.044 & 14.49 & 9 & 24.31 & 131.0 & $2^{a}$ & 0.09 & 22.29 \\
\hline $1218+304$ & $\ldots$ & 0.130 & 15.80 & 6 & 22.79 & 62.0 & 5 & $>1.34$ & 22.72 \\
\hline $1219+285$ & ON 231 & 0.102 & 15.40 & 7 & 23.07 & 2058.0 & $2^{a}$ & 3.25 & 22.67 \\
\hline $1221+248$ & $\ldots$ & 0.218 & 17.65 & 1 & 23.64 & 27.85 & 2 & 1.53 & 22.45 \\
\hline $1229+645$ & $\ldots$ & 0.164 & 16.89 & 1 & 23.73 & 42.49 & 2 & 1.34 & 22.49 \\
\hline $1235+632$ & $\ldots$ & 0.297 & 18.59 & 1 & 24.00 & 13.0 & 5 & $>0.32$ & 22.35 \\
\hline $1400+162$ & $\ldots$ & 0.244 & 16.74 & 9 & 26.27 & 233.0 & $2^{a}$ & -0.07 & 22.91 \\
\hline $1402+042$ & $\ldots$ & 0.344 & 16.88 & 8 & 24.40 & 21.43 & 2 & 0.53 & 23.17 \\
\hline $1407+599$ & $\ldots$ & 0.495 & 19.67 & 1 & 25.76 & 14.12 & 2 & 0.08 & 22.39 \\
\hline $1418+546$ & $\ldots$ & 0.152 & 15.39 & 6 & 24.31 & 1058.0 & $2^{a}$ & 2.09 & 23.03 \\
\hline $1426+427$ & $\ldots$ & 0.130 & 16.40 & 9 & 23.50 & 31.0 & 5 & $>1.04$ & 22.48 \\
\hline $1443+638$ & $\ldots$ & 0.299 & 19.65 & 1 & 24.63 & 8.36 & 2 & 0.33 & 21.93 \\
\hline $1458+228$ & $\ldots$ & 0.235 & 16.79 & 1 & 23.98 & 29.0 & 2 & 1.28 & 22.86 \\
\hline $1514-241$ & AP Lib & 0.049 & 14.97 & 7 & 23.61 & 2562.0 & $2^{a}$ & 2.18 & 22.19 \\
\hline $1534+018$ & $\ldots$ & 0.312 & 18.70 & 1 & 25.28 & 28.84 & 2 & 0.26 & 22.35 \\
\hline $1538+149$ & 4C 14.60 & 0.605 & 17.89 & 6 & 26.94 & 1337.0 & $2^{a}$ & 1.17 & 23.29 \\
\hline $1552+203$ & $\ldots$ & 0.222 & 17.70 & 1 & 24.65 & 33.09 & 2 & 0.61 & 22.44 \\
\hline $1652+398$ & Mrk501 & 0.034 & 14.08 & 6 & 23.52 & 1376.0 & $2^{a}$ & 1.66 & 22.23 \\
\hline $1727+502$ & $\ldots$ & 0.055 & 16.12 & 6 & 23.83 & 175.0 & $2^{a}$ & 0.89 & 21.83 \\
\hline $1749+096$ & $\ldots$ & 0.320 & 17.32 & 6 & 23.89 & 744.0 & $2^{a}$ & $>3.11$ & 22.93 \\
\hline $1807+698$ & $3 \mathrm{C} 371$ & 0.050 & 14.57 & 7 & 25.04 & 1350.0 & $2^{a}$ & 0.48 & 22.37 \\
\hline $2143+070$ & $\ldots$ & 0.237 & 18.04 & 1 & 24.99 & 44.63 & 2 & 0.46 & 22.37 \\
\hline $2155-304$ & $\cdots$ & 0.117 & 13.31 & 6 & 25.18 & 252.00 & $2^{a}$ & 0.37 & 23.62 \\
\hline $2200+420$ & BL Lac & 0.069 & 15.42 & 7 & 23.93 & 3310.00 & $2^{a}$ & 2.26 & 22.31 \\
\hline $2201+044$ & $\ldots$ & 0.028 & 15.47 & 8 & 23.70 & 316.00 & 5 & 0.25 & 21.50 \\
\hline $2254+074$ & $\ldots$ & 0.190 & 16.29 & 6 & 24.51 & 454.00 & $2^{a}$ & 1.73 & 22.87 \\
\hline $2356-309$ & $\ldots$ & 0.165 & 17.18 & 8 & 23.50 & 42.00 & 5 & 0.90 & 22.38 \\
\hline
\end{tabular}

Redshift with a star is from Rector \& Stocke (2001) $-\log R_{\mathrm{c}}^{\dagger}$ was thus calculated for this new $z$ using the $\log R_{\mathrm{c}}$ quoted in Vermeulen \& Cohen (1994). References: (1): Morris et al. (1991); (2): Perlman \& Stocke (1993) (EMSS XBLs except $2^{a}$ which are RBLs, $1.4 \mathrm{GHz}$ ); (4): log $R_{\mathrm{c}}$ from Vermeulen \& Cohen (1994); (5): Laurent-Muehleisen et al. (1993) (HEAO-1 XBLs, $1.5 \mathrm{GHz}$ ); (6): Pica et al. (1988); (7): Webb et al. (1988); (8): Falomo et al. (1994); (9): Padovani \& Giommi (1995). 
P. Kharb and P. Shastri: Optical nuclei and the F-R Divide, Online Material p 5

Table C.4. The Radio-loud quasars.

\begin{tabular}{|c|c|c|c|c|c|c|c|c|c|}
\hline $\begin{array}{l}\text { IAU } \\
\text { name }\end{array}$ & $\begin{array}{c}\text { Alternate } \\
\text { name }\end{array}$ & $\begin{array}{c}\text { Redshift } \\
z\end{array}$ & $m_{v}$ & Ref. & $\begin{array}{l}\log L_{\text {ext }} \\
\mathrm{W} \mathrm{Hz} z^{-1}\end{array}$ & $\begin{array}{c}S_{\mathrm{c}}(5 \mathrm{GHz}) \\
\mathrm{mJy}\end{array}$ & Ref. & $\log R_{\mathrm{c}}$ & $\begin{array}{c}\log L_{\mathrm{o}} \\
\mathrm{W} \mathrm{Hz}{ }^{-1}\end{array}$ \\
\hline $0016+731$ & $\ldots$ & 1.781 & 19.00 & 4 & 27.37 & $>1500.0$ & 1 & $>0.7$ & 23.88 \\
\hline $0106+013$ & $\ldots$ & 2.107 & 18.39 & 4 & 27.65 & 3470.0 & 1 & 0.9 & 24.29 \\
\hline $0153+744$ & $\ldots$ & 2.338 & 16.00 & 4 & 26.26 & 1510.0 & 1 & $>2.0$ & 25.35 \\
\hline $0212+735$ & $\ldots$ & 2.367 & 20.00 & 4 & 25.03 & 2200.0 & 1 & $>3.4$ & 23.76 \\
\hline $0234+285$ & $\ldots$ & 1.207 & 18.50 & 4 & 25.68 & 1440.0 & 1 & 2.1 & 23.71 \\
\hline $0333+321$ & NRAO140 & 1.259 & 17.50 & 4 & 26.44 & 2460.0 & 1 & 1.6 & 24.15 \\
\hline 0458-020 & $\ldots$ & 2.286 & 19.50 & 4 & 27.17 & 1600.0 & 1 & 1.1 & 23.93 \\
\hline $0615+820$ & $\ldots$ & 0.710 & 17.50 & 4 & 26.38 & $>900.0$ & 1 & $>0.8$ & 23.60 \\
\hline $0711+356$ & $\ldots$ & 1.620 & 19.00 & 4 & 25.96 & 1500.0 & 1 & $>2.1$ & 23.79 \\
\hline $0723+679$ & $3 \mathrm{C} 179$ & 0.846 & 18.00 & 4 & 27.54 & 320.0 & 1 & -0.68 & 23.57 \\
\hline $0835+580$ & $3 \mathrm{C} 205$ & 1.536 & 17.62 & 4 & 27.89 & 23.0 & 1 & -1.74 & 24.29 \\
\hline $0836+710$ & $\ldots$ & 2.180 & 16.50 & 4 & 27.04 & 2550.0 & 1 & 1.4 & 25.08 \\
\hline $0839+616$ & $\ldots$ & 0.862 & 17.85 & 4 & 26.84 & 34.0 & 1 & -0.94 & 23.64 \\
\hline $0850+581$ & $\ldots$ & 1.322 & 18.00 & 4 & 27.45 & 1090.0 & 1 & 0.27 & 23.99 \\
\hline $0906+430$ & $3 \mathrm{C} 216$ & 0.670 & 18.10 & 4 & 27.21 & 1060.0 & 1 & -0.01 & 23.30 \\
\hline $0923+392$ & 4C 39.25 & 0.698 & 17.86 & 4 & 26.77 & 7320.0 & 1 & 1.3 & 23.44 \\
\hline $1039+811$ & $\ldots$ & 1.260 & 16.50 & 4 & 26.20 & 1120.0 & 1 & 1.5 & 24.55 \\
\hline $1040+123$ & $3 \mathrm{C} 245$ & 1.028 & 17.29 & 4 & 27.44 & 860.0 & 1 & 0.0 & 24.04 \\
\hline $1150+812$ & $\ldots$ & 1.250 & 18.50 & 4 & 26.50 & 1140.0 & 1 & 1.2 & 23.74 \\
\hline $1156+295$ & $\ldots$ & 0.729 & 14.41 & 4 & 26.32 & 810.0 & 1 & 0.83 & 24.86 \\
\hline $1222+216$ & 4C 21.35 & 0.435 & 17.50 & 4 & 26.47 & 420.0 & 1 & -0.01 & 23.14 \\
\hline $1226+023$ & $3 \mathrm{C} 273$ & 0.158 & 12.85 & 4 & 26.70 & 39000.0 & 1 & 0.9 & 24.08 \\
\hline $1253-055$ & $3 \mathrm{C} 279$ & 0.538 & 17.75 & 4 & 27.07 & 14500.0 & 1 & 1.1 & 23.24 \\
\hline $1458+718$ & $3 \mathrm{C} 309.1$ & 0.905 & 16.78 & 4 & 27.63 & 2680.0 & 1 & 0.2 & 24.12 \\
\hline $1641+399$ & $3 \mathrm{C} 345$ & 0.594 & 15.96 & 4 & 26.33 & 5520.0 & 1 & 1.5 & 24.05 \\
\hline $1642+690$ & $\ldots$ & 0.751 & 20.50 & 4 & 26.67 & 1260.0 & 1 & 0.7 & 22.45 \\
\hline $1721+343$ & 4C 34.47 & 0.206 & 15.46 & 4 & 25.95 & 470.0 & 1 & -0.05 & 23.27 \\
\hline $1828+487$ & $3 \mathrm{C} 380$ & 0.691 & 16.81 & 4 & 27.32 & 6590.0 & 1 & 0.7 & 23.85 \\
\hline $1830+285$ & $4 C 28.45$ & 0.594 & 17.16 & 4 & 27.02 & 450.0 & 1 & -0.28 & 23.57 \\
\hline $1901+319$ & $3 C 395$ & 0.635 & 17.50 & 4 & 26.81 & 1480.0 & 1 & 0.5 & 23.49 \\
\hline $1928+738$ & $\ldots$ & 0.302 & 16.06 & 4 & 25.75 & 3210.0 & 1 & 1.3 & 23.38 \\
\hline $1951+498$ & $\ldots$ & 0.466 & 17.50 & 4 & 26.09 & 91.0 & 1 & -0.24 & 23.20 \\
\hline $2223-052$ & $3 \mathrm{C} 446$ & 1.404 & 18.39 & 4 & 28.24 & 2310.0 & 1 & -0.15 & 23.90 \\
\hline $2251+158$ & $3 \mathrm{C} 454.3$ & 0.859 & 16.10 & 4 & 27.05 & 9690.0 & 1 & 1.3 & 24.34 \\
\hline
\end{tabular}

References: (1): $\log R_{\mathrm{c}}$ from Vermeulen \& Cohen (1994); (4): Véron-Cetty \& Véron (1998). 